\title{
DIAGNÓSTICO SITUACIONAL DE UMA CLÍNICA DE TRATAMENTO ONCOLÓGICO DE BELO HORIZONTE
}

\section{SITUATIONAL DIAGNOSIS OF A BELO HORIZONTE ONCOLOGICAL TREATMENT CLINIC}

Alice Edwirges de Castro Andrade ${ }^{1}$, Daniel dos Santos Fernandes², Aline Corrêa de Almeida ${ }^{3}$, Eliada Neves de Queiroz Abreu ${ }^{4}$, Nayara Pereira Mota ${ }^{5}$, Nicoly Cirino de Rezende ${ }^{6}$, Tailane de Jesus Santos ${ }^{7}$

\author{
Submetido em: 05/04/2021 \\ Aprovado em: 26/04/2021
}

\section{RESUMO}

Introdução: O diagnóstico situacional é uma ferramenta de coleta, descrição e análise de dados, que permite o conhecimento de pontos de melhoria em uma instituição de saúde. Com o diagnóstico situacional é possível criar intervenções focadas nos problemas encontrados durante o estudo. Metodologia: Trata-se de uma pesquisa de campo descritiva realizada em uma clínica oncológica de Belo Horizonte. Resultados: Durante o estudo, foi avaliada a estrutura organizacional, recursos físicos, humanos e materiais, dos instrumentos gerenciais e a definição dos indicadores assistenciais de enfermagem. Todos os dados coletados foram descritos e comparados à literatura e normas vigentes. Conclusão: Com o estudo, foi possível identificar divergências positivas e negativas entre a clínica e as normas, porém as positivas se sobressaíram. Além disso, a elaboração do Diagnóstico Situacional proporciona ao acadêmico a capacidade de pesquisar, analisar dados e de desenvolver um senso crítico em relação às instituições de saúde e o serviço oferecido por elas.

PALAVRAS-CHAVE: Diagnóstico situacional; Gestão em saúde; Enfermagem.

\begin{abstract}
Introduction: Situational diagnosis is a tool for collecting, describing and analyzing data, which allows knowledge of points of improvement in a health institution. With the situational diagnosis, it is possible to create interventions focused on the problems encountered during the study. Methodology: This is a descriptive field research carried out in an oncology clinic in Belo Horizonte. Results: During the study, the organizational structure, physical, human and material resources of the management instruments and the definition of nursing care indicators were evaluated. All data collected were described and compared to the current literature and standards. Conclusion: With the study, it was possible to identify positive and negative divergences between the clinic and the norms, but the positive ones stood out. In addition, the preparation of the Situational Diagnosis provides the academic with the ability to research, analyze data and develop a critical sense in relation to health institutions and the service offered by them.
\end{abstract}

KEYWORDS: Situational diagnosis; Health management; Nursing.

\footnotetext{
${ }^{1}$ Acadêmica de Enfermagem na Universidade Vale do Rio Verde, campus Belo Horizonte.

2 Enfermeiro mestre em Medicina e Biomedicina. Professor e coordenador do curso de Enfermagem na Universidade Vale do Rio Verde, campus Belo Horizonte.

${ }^{3}$ Acadêmica de Enfermagem na Universidade Vale do Rio Verde, campus Belo Horizonte.

${ }^{4}$ Acadêmica de Enfermagem na Universidade Vale do Rio Verde, campus Belo Horizonte.

${ }^{5}$ Acadêmica de Enfermagem na Universidade Vale do Rio Verde, campus Belo Horizonte.

${ }^{6}$ Acadêmica de Enfermagem na Universidade Vale do Rio Verde, campus Belo Horizonte.

7 Acadêmica de Enfermagem na Universidade Vale do Rio Verde, campus Belo Horizonte.
} 


\section{RECIMA21 - REVISTA CIENTÍFICA MULTIDISCIPLINAR}

DIAGNÓSTICO SITUACIONAL DE UMA CLÍNICA DE TRATAMENTO ONCOLÓGICO DE BELO HORIZONTE Alice Edwirges de Castro Andrade, Daniel dos Santos Fernandes, Aline Corrêa de Almeida, Eliada Neves de Queiroz Abreu, Nayara Pereira Mota, Nicoly Cirino de Rezende, Tailane de Jesus Santos

\section{INTRODUÇÃO}

As ações em saúde devem ser planejadas e direcionadas e, para isso, é necessário conhecer a dinâmica, realidade, riscos e potencialidades da população inserida no serviço. Para isso, é fundamental compreender todo o serviço de saúde, desde a organização e rotina ao serviço prestado (COREN, 2020).

O Diagnóstico Situacional é uma ferramenta que permite conhecer os problemas e as necessidades de um serviço de saúde, sendo de fundamental importância para o levantamento de dados, análise potencialidades e problemas encontrados, fornecendo, assim, recursos para um planejamento estratégico situacional. É através desse planejamento estratégico situacional que há a possibilidade de desenvolver ações de saúde focadas para os pontos de melhoria do serviço (SANTOS, 2010).

Sendo assim, o diagnóstico situacional é fundamental para o levantamento de pontos de melhoria, que fundamentarão o planejamento estratégico situacional, permitindo desenvolver ações mais assertivas e focadas nos problemas encontrados.

Tendo em vista a importância do diagnóstico situacional de uma instituição, acredita-se que o desenvolvimento de habilidades e competências de acadêmicos de enfermagem em torno da realização do diagnóstico situacional seja fundamental para a formação profissional do enfermeiro.

\section{OBJETIVOS}

Objetivo Geral

- Realizar o Diagnóstico Situacional de uma clínica de tratamento oncológico de Belo Horizonte/MG.

Objetivos Específicos

- Reconhecer a estrutura organizacional;

- Avaliar os recursos físicos e infraestrutura;

- Avaliar os recursos humanos;

- Avaliar os recursos materiais;

- Identificar instrumentos gerenciais utilizados;

- Identificar e definir indicadores assistenciais de enfermagem;

- Identificar potencialidades e melhorias a serem feitas na instituição. 


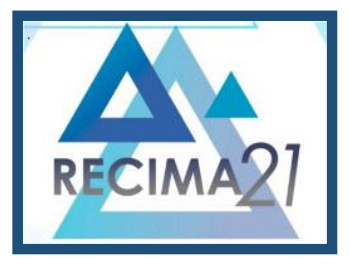

\section{RECIMA21 - REVISTA CIENTÍFICA MULTIDISCIPLINAR}

DIAGNÓSTICO SITUACIONAL DE UMA CLÍNICA DE TRATAMENTO ONCOLÓGICO DE BELO HORIZONTE Alice Edwirges de Castro Andrade, Daniel dos Santos Fernandes, Aline Corrêa de Almeida, Eliada Neves de Queiroz Abreu, Nayara Pereira Mota, Nicoly Cirino de Rezende, Tailane de Jesus Santos

\section{METODOLOGIA}

- Tipo de Estudo

Trata-se de um estudo descritivo, exploratório do tipo relato de experiência o qual será apresentado em forma de Relatório de Diagnóstico Situacional.

- Local do Estudo

A instituição foco do estudo é uma clínica especializada na prevenção e tratamento de doenças neoplásicas de Belo Horizonte/MG. Foi fundada em 1994 e conta com as áreas de oncologia, hematologia, clínica da dor, oncogenética, além da equipe multidisciplinar composta por nutricionistas, psicólogos, dentistas, acupunturistas e enfermeiros.

\section{- Etapas e Procedimentos}

$1^{\text {a }}$ etapa: Visita técnica à instituição no dia 29 de outubro de 2020. Foi possível conhecer os espaços assistenciais e administrativos da empresa. Foi realizada uma reunião com a enfermeira responsável Mariana Assunção, que explicou todo o processo do Diagnóstico Situacional e elencou os principais pontos a serem abordados de acordo com a realidade da instituição.

$2^{2}$ etapa: Foi realizada uma reunião virtual entre alguns integrantes do grupo e a enfermeira Referência Técnica (RT) para conhecimento e análise da estrutura organizacional e infraestrutura da empresa.

$3^{\text {a }}$ etapa: Disponibilização, análise e compilação dos dados fornecidos pela instituição por meio da enfermeira RT.

4ª etapa: Análise dos dados obtidos com os dados contidos na literatura.

\section{RESULTADOS E DISCUSSÃO}

\section{RECONHECIMENTO DA ESTRUTURA ORGANIZACIONAL DO SERVIÇO}

Na lei 7.498/86, que dispõe sobre a regulamentação do exercício de enfermagem, nota-se muitas diferenças entre cada profissional, tendo cada um suas atuações diferenciadas dentro da unidade de saúde. O enfermeiro encontra-se com um campo de atuação mais amplo, atuando em todos os níveis de atendimento, sendo também capacitado para tomar decisões imediatas em casos de emergência. Trabalha, também, em organizações dos serviços de enfermagem e supervisiona técnicos e auxiliares. Técnicos atuam em grau auxiliar de cuidados ao paciente, sendo capacitado para cumprir procedimentos em casos de média e alta complexidade, como atendimento ao paciente de pré e pós-operatório. Os auxiliares trabalham com procedimentos mais simples, em setores ambulatoriais, executam ações de menor complexidade, podendo fazer curativos, aplicações de vacinas e até administração de medicamentos, porém em casos de baixa gravidade. 


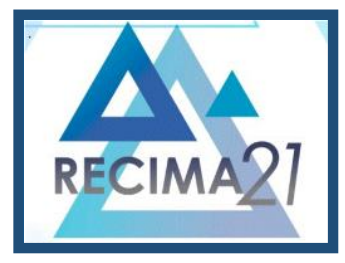

\section{RECIMA21 - REVISTA CIENTÍFICA MULTIDISCIPLINAR}

DIAGNÓSTICO SITUACIONAL DE UMA CLíNICA DE TRATAMENTO ONCOLÓGICO DE BELO HORIZONTE Alice Edwirges de Castro Andrade, Daniel dos Santos Fernandes, Aline Corrêa de Almeida, Eliada Neves de Queiroz Abreu, Nayara Pereira Mota, Nicoly Cirino de Rezende, Tailane de Jesus Santos

$\mathrm{Na}$ enfermagem oncológica, o enfermeiro deve sempre permanecer ao lado do paciente, em qualquer momento. A enfermagem oncológica tem como objetivo a promoção da qualidade de vida do paciente, intervindo e avaliando na sua saúde física e mental.

O serviço oncológico conta com o apoio de uma equipe multidisciplinar composta por diversos especialistas, como psicólogos, nutricionistas, dentistas, fisioterapeutas, farmacêuticos, entre outros. Cada um sendo responsável por diferentes cuidados ao paciente.

Sendo assim, todas as posições, cargos e funções prestam atendimentos visando a condição do paciente, contribuindo para a segurança do paciente, fortalecendo a própria equipe, trazendo ordem nas relações de trabalho. Uma observação de um determinado especialista pode alertar o outro, fazendo com que os profissionais contribuam significativamente com o trabalho do outro (MUFASA, 2020).

Para estabelecer a organização de uma empresa, o organograma representa a estrutura organizacional, a qual mostra graficamente como estão dispostas as unidades funcionais, a hierarquia, ou seja, quem é chefe de quem, e as relações de comunicação existentes dentro da organização. Também tem a capacidade de apresentar a distribuição de cargos e de autoridade (COFEN, 2020).

A fig. 1 demonstra o organograma institucional da clínica. Nele podemos observar que em seu topo encontram-se as posições hierárquicas mais altas da empresa, vindo em seguida as mais básicas e operacionais, facilitando assim as interações de tais hierarquias e também integração de áreas e cargos.

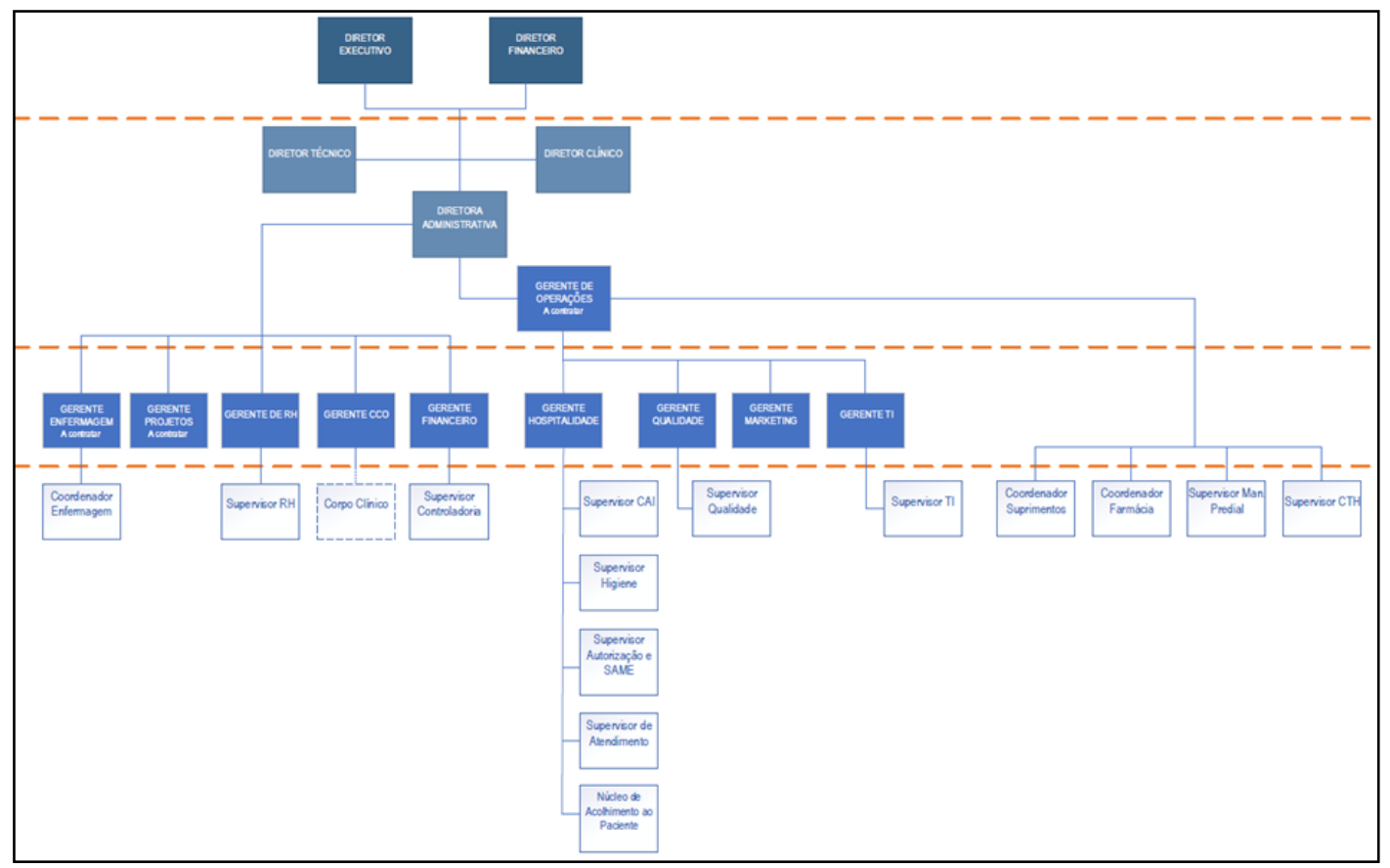

Fig. 1: Organograma institucional. Fonte: Cedido pela instituição. 


\section{RECIMA21 - REVISTA CIENTÍFICA MULTIDISCIPLINAR}

DIAGNÓSTICO SITUACIONAL DE UMA CLÍNICA DE TRATAMENTO ONCOLÓGICO DE BELO HORIZONTE Alice Edwirges de Castro Andrade, Daniel dos Santos Fernandes, Aline Corrêa de Almeida, Eliada Neves de Queiroz Abreu, Nayara Pereira Mota, Nicoly Cirino de Rezende, Tailane de Jesus Santos

Além do organograma é essencial que se descreva as funções de cada cargo. Entende-se que cada função requer habilidades e competências diferentes e que todas somam-se na busca da manutenção da missão e na defesa dos valores institucionais.

\section{AVALIAÇÃO DOS RECURSOS FÍSICOS E INFRAESTRUTURA}

Segundo Amorin et al. (2013), a avaliação da estrutura física das unidades de saúde compreende as áreas internas e externas que a compõem e está vinculada à funcionalidade. Os aspectos físicos dos Estabelecimentos Assistenciais de Saúde (EAS) estão relacionados com os processos de arquitetura e engenharia e têm objetivos voltados para a promoção, prevenção e recuperação de saúde.

De acordo com Moura et. al., (2010), a estrutura física define quais recursos são necessários para proporcionar a realização dos atendimentos de saúde. Estes recursos envolvem a análise de instalação e equipamentos, que inclui o prédio e os seus componentes físicos. Segundo os autores, a estrutura física das instituições de saúde deve conter espaços que possa atender todas as necessidades assistenciais da comunidade

Amorin et. al., (2013) afirmam que os estabelecimentos assistenciais de saúde têm grande impacto na saúde e segurança dos pacientes e é função do enfermeiro conhecer a área física das EAS para garantir uma boa qualidade na assistência. Cabe a gestão de enfermagem ter o domínio da complexidade do edifício e está atento aos reparos necessários para manter a estrutura física dentro das leis vigentes.

Diante disto, no dia 29 de outubro de 2020 foi realizada uma visita técnica pelas acadêmicas de enfermagem da Unincor-BH na clínica, em Belo Horizonte/ MG para buscar informações sobre o funcionamento ambulatorial da clínica. A instituição tem uma unidade e mais três anexos compostos atendimento ambulatorial, atendimento para medicações via oral e equipe multidisciplinar.

Deste modo, o local deste estudo apresenta a seguinte estrutura física distribuída em um edifico de 6 andares:

- $\quad 1^{\circ}$ andar: 2 recepções, 4 consultórios, 1 recepção de apoio, concierge com 1 sala, 4 mesas e uma entrada ao fundo para sala da central de atendimentos;

- Sobreloja: 1 recepção, consultórios, financeiros, sênior, sala da diretoria, sanitários, vestiário, copa e sala de hospitalidade;

- $2^{\circ}$ andar (ambulatório): 1 posto de enfermagem com 5 camas e 8 poltronas; 1 posto de enfermagem com 4 poltronas, farmácia com cabine de segurança biológica, recepção, sanitários, copa, DML e expurgo; 


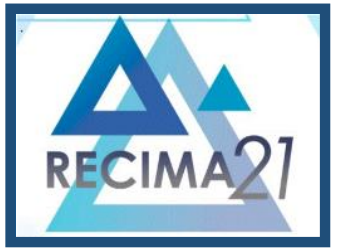

\section{RECIMA21 - REVISTA CIENTÍFICA MULTIDISCIPLINAR}

DIAGNÓSTICO SITUACIONAL DE UMA CLÍNICA DE TRATAMENTO ONCOLÓGICO DE BELO HORIZONTE Alice Edwirges de Castro Andrade, Daniel dos Santos Fernandes, Aline Corrêa de Almeida, Eliada Neves de Queiroz Abreu, Nayara Pereira Mota, Nicoly Cirino de Rezende, Tailane de Jesus Santos

- $3^{\circ}$ andar: adaptado para pacientes que irão tomar medicações por injeção, composto por 1 recepção, 3 poltronas, 1 cama, sanitários e auditório.

- $4^{\circ}$ andar: ambulatório com 9 camas, posto de enfermagem, sanitários, sala de utilidades, DML e sala de coordenação de enfermagem;

- $5^{\circ}$ e $6^{\circ}$ andar: recepção de apoio e 4 consultórios em cada andar.

As figuras de 2 a 7 demonstram como é o dimensionamento de cada um destes espaços, representando a planta em 3D do EAS foco deste diagnóstico:

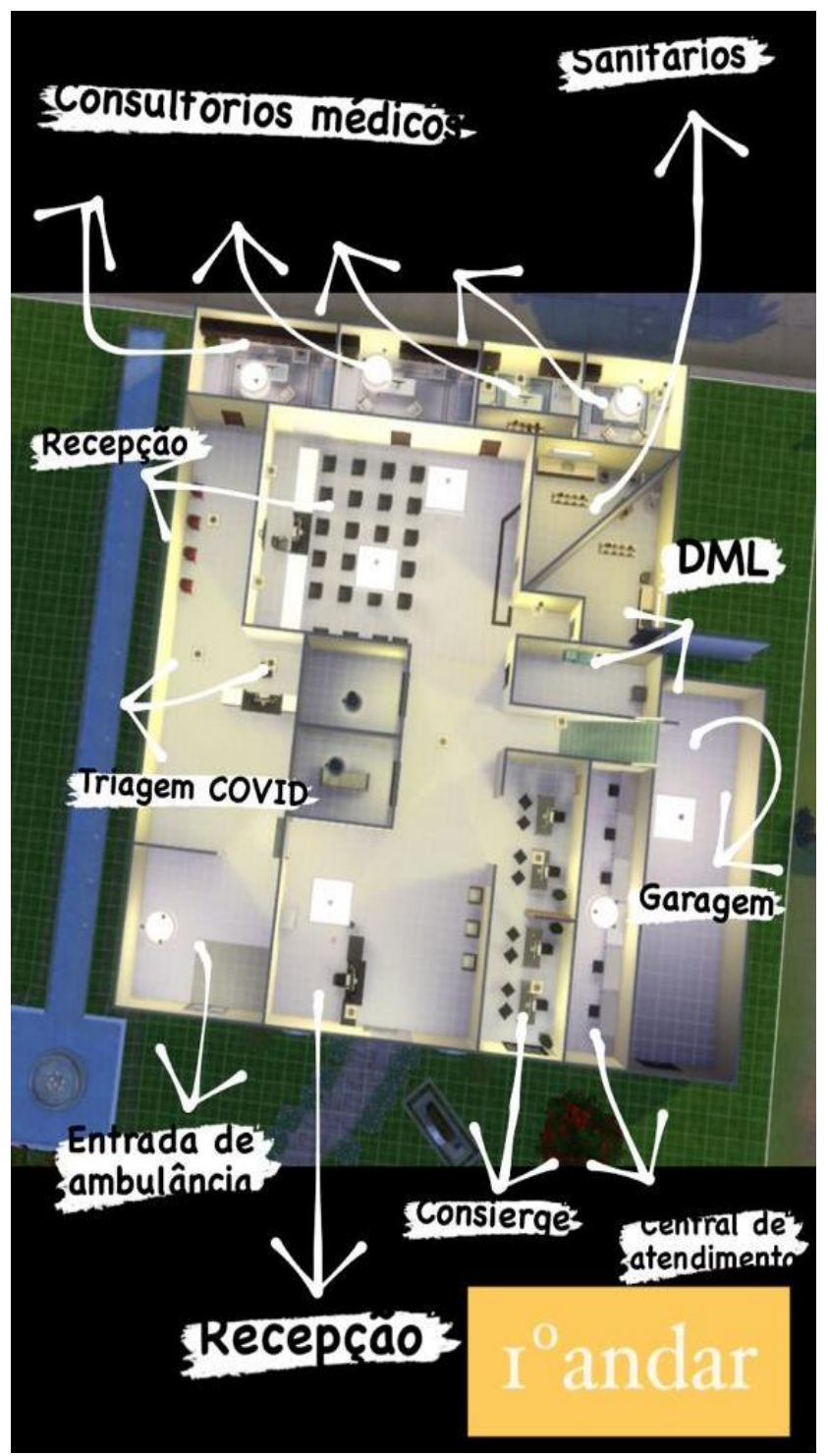

Fig. 2: Planta institucional. Fonte: Criação dos autores. autores.

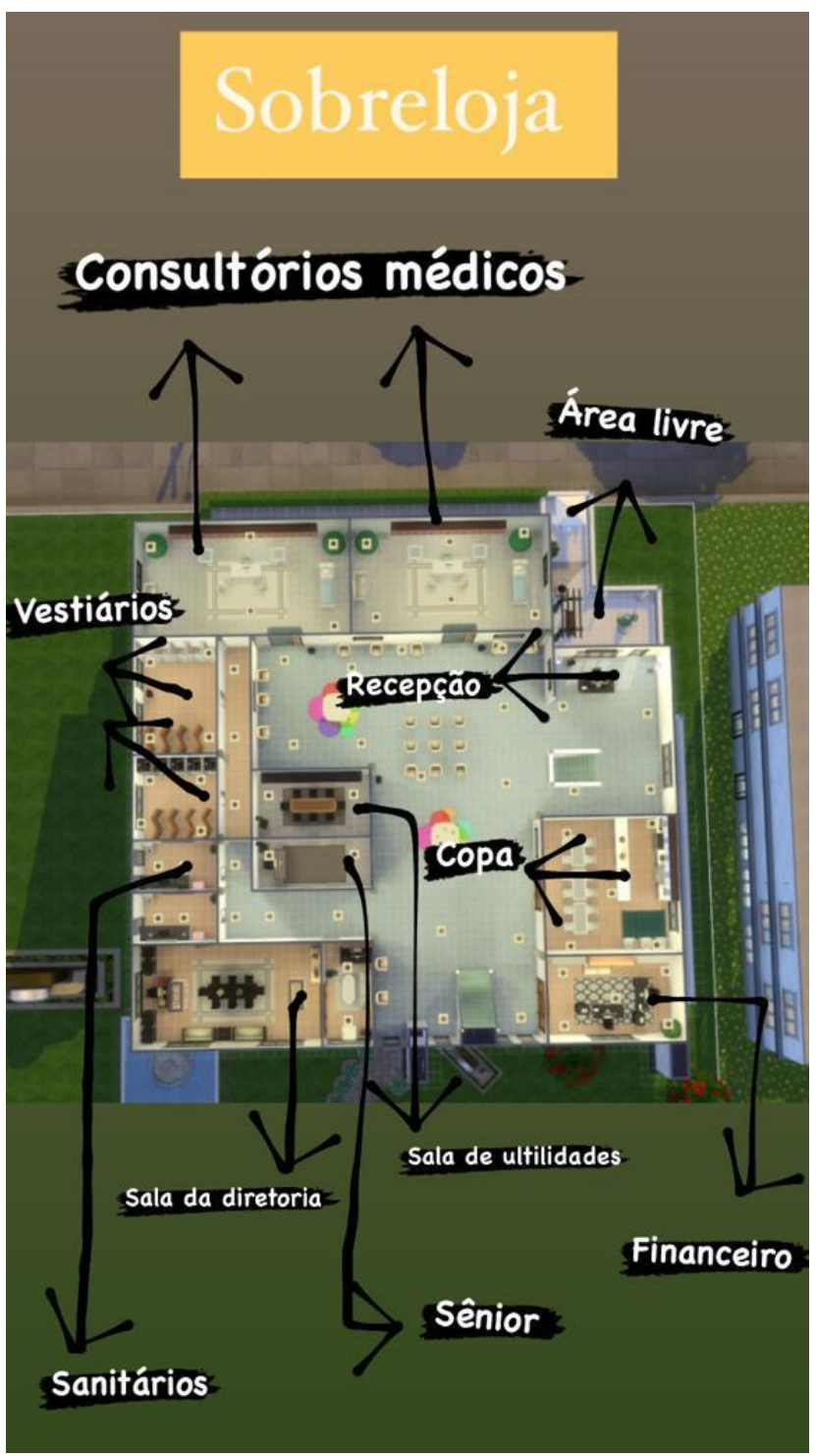

Fig. 3: Planta institucional. Fonte: Criação dos autores. 


\section{RECIMA21 - REVISTA CIENTÍFICA MULTIDISCIPLINAR}

DIAGNÓSTICO SITUACIONAL DE UMA CLíNICA DE TRATAMENTO ONCOLÓGICO DE BELO HORIZONTE Alice Edwirges de Castro Andrade, Daniel dos Santos Fernandes, Aline Corrêa de Almeida, Eliada Neves de Queiroz Abreu, Nayara Pereira Mota, Nicoly Cirino de Rezende, Tailane de Jesus Santos

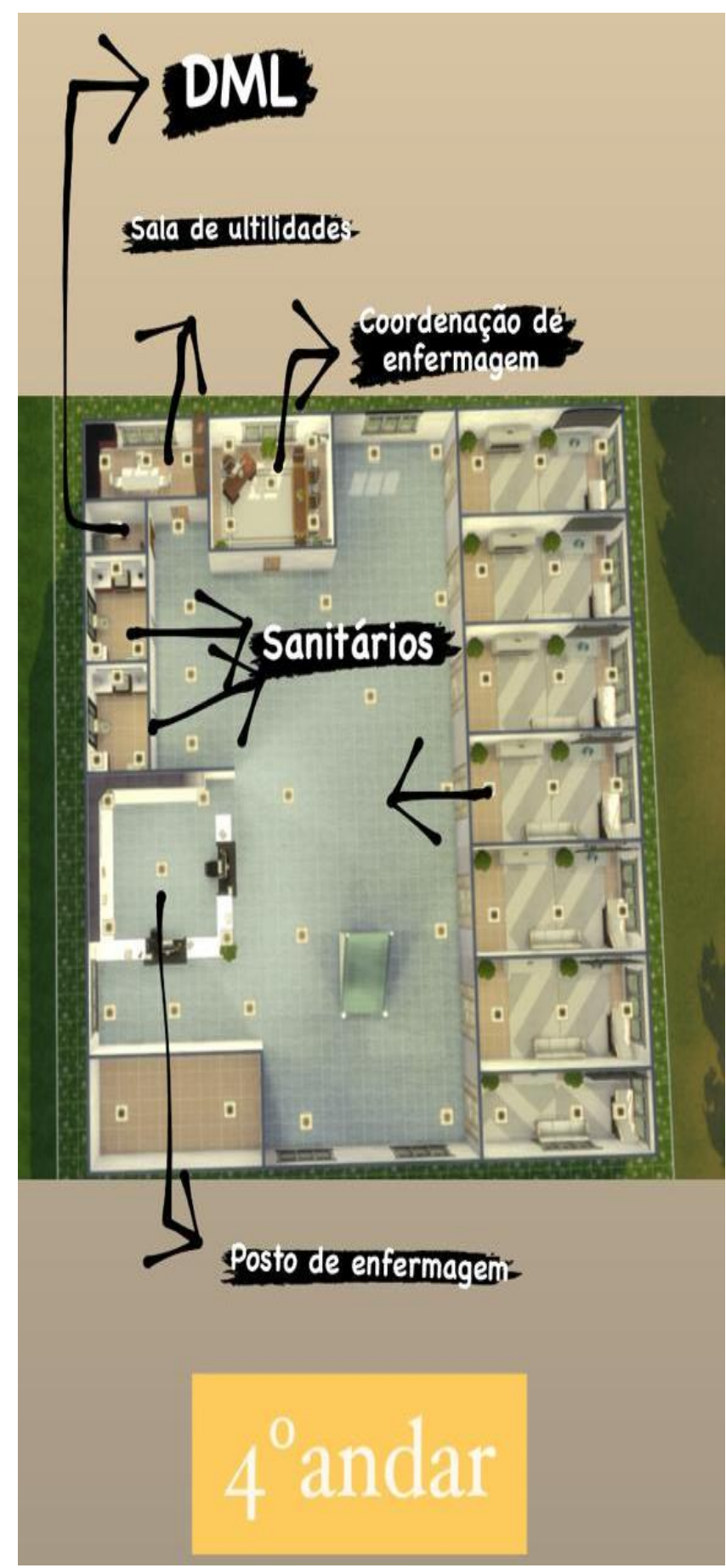

Fig. 6: Planta institucional. Fonte: Criação dos autores.

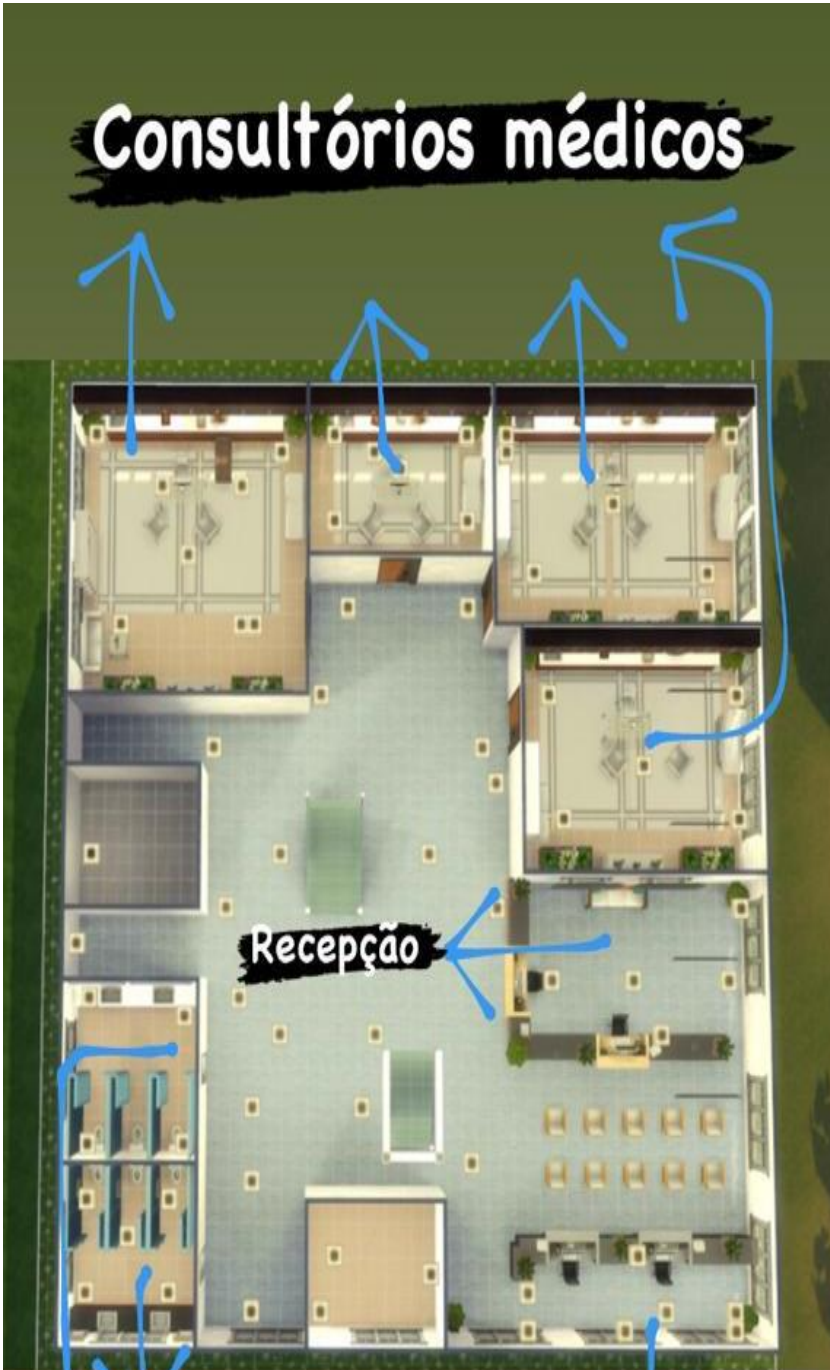

\section{Sanitarios}

Recepção de apoio=

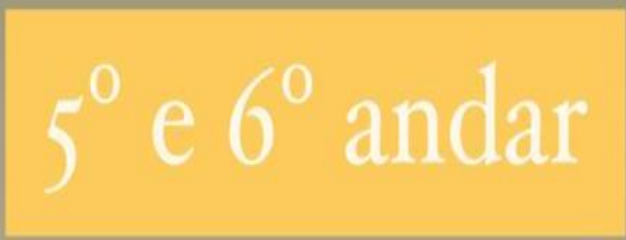

Fig. 7: Planta institucional. Fonte: Criação dos autores. 


\section{RECIMA21 - REVISTA CIENTÍFICA MULTIDISCIPLINAR}

DIAGNÓSTICO SITUACIONAL DE UMA CLíNICA DE TRATAMENTO ONCOLÓGICO DE BELO HORIZONTE Alice Edwirges de Castro Andrade, Daniel dos Santos Fernandes, Aline Corrêa de Almeida, Eliada Neves de Queiroz Abreu, Nayara Pereira Mota, Nicoly Cirino de Rezende, Tailane de Jesus Santos

Corroborando com esta discussão, encontra-se a RDC n 50 de 21 de fevereiro de 2002, que dispõe sobre o regulamento técnico para o planejamento, programação, elaboração e avaliação dos projetos físicos dos estabelecimentos assistenciais de saúde. Todas os EAS são obrigatoriamente elaborados por meio desta RDC. Esta resolução descreve que para a elaborar um projeto físico de uma EAS é necessário descrever o conjunto e o número de edificações, número de pavimentos, esquema de infraestrutura e serviço, dimensões, número de leitos, localização de sanitários, farmácia, DML, instalações radioativas e expurgo.

Para a execução de qualquer estrutura física de um EAS é exigido a avaliação do projeto arquitetônico em questão pela Vigilância Sanitária, que emitirá a licença para sua execução de acordo com o que está descrito no inciso II do Art. 10 e 14 da Lei 643/77 que dispõe das penalidades para quem cometer infração contra a RDC 50/2002. A construção dos estabelecimentos assistenciais de saúde também precisa estar baseada na Constituição Federal de 1988 e na Lei Orgânica de Saúde 8080/90.

De acordo com a RDC 50/2002, os estabelecimentos assistenciais de saúde especializados em atendimentos ambulatoriais de quimioterapia são unidades funcionais que necessitam de condições especiais para sua funcionalidade devido abrigarem equipamentos geradores de calor e gases não tóxicos.

A RDC 50/2002 descreve que uma unidade funcional de diagnóstico e terapia deve ser construída em conformidade com a Portaria MS no 3.535 de 02/09/98 e Portaria MS 255 de 31/03/99, que define os critérios para conformidade dos centros especializados em oncologia. Estes ambientes devem conter consultórios médicos, sala para aplicação de quimioterápicos, área para guardar materiais e medicamentos, postos de enfermagem; salas de espera de pacientes, sala de utilidades, sanitários, copa, sala administrativa e farmácia.

Deste modo, os centros especializados em terapias antineoplásicas devem respeitar as instalações indicadas na figura 8:

\begin{tabular}{|c|c|c|c|c|}
\hline \multicolumn{5}{|c|}{ CIONAL: 4 - APOIO AO DIAGNOSTICO E TERAPIA (cont) } \\
\hline \multirow[t]{2}{*}{$\overline{N^{N} \text { ATIV. }}$} & \multirow[t]{2}{*}{ UNIDADE/AMBIENTE } & \multicolumn{2}{|c|}{ DIMENSIONAMENTO } & \multirow[t]{2}{*}{ INSTALAÇÓES } \\
\hline & & QUANTIFICACCÃO (min.) & DIMENSÃO(min.) & \\
\hline 4.11 & Ouimioterapia & & & \\
\hline \begin{tabular}{|l|l|l|}
4.11 .1 \\
\end{tabular} & Consultónio indiferenciado & 1. 'Tn loco" ou nฐ̃o & $7,5 \mathrm{~m}^{2}$ & $\mathrm{HF}$ \\
\hline $4.11 .4 ; 4.115$ & 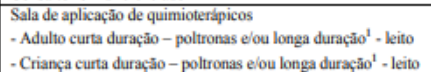 & $\begin{array}{l}\text { 1. No caso de haver atendimento } \\
\text { pediatrico, a sala deve ser exclusiva }\end{array}$ & $7,0 \mathrm{~m}^{2}$ por leito e $5,0 \mathrm{~m}^{2}$ por poltrona & HF,FO,FAM;EE \\
\hline 4.11 .4 & Ára de material e medicamentos ${ }^{2}$ & $\begin{array}{l}\text { 1, quando o preparo das drogas fox } \\
\text { feito na farmscia }\end{array}$ & $3,0 \mathrm{~m}^{2}$ & \\
\hline $4.1113 ; 4.11 .6$ & Posto de enfermagem e servipos & $\begin{array}{l}1 \text { a cada } 12 \text { poltronas/eitos ou } \\
\text { fraça }\end{array}$ & $6,0 \mathrm{~m}^{2}$ & $\mathrm{HF}$ \\
\hline \multicolumn{5}{|c|}{ 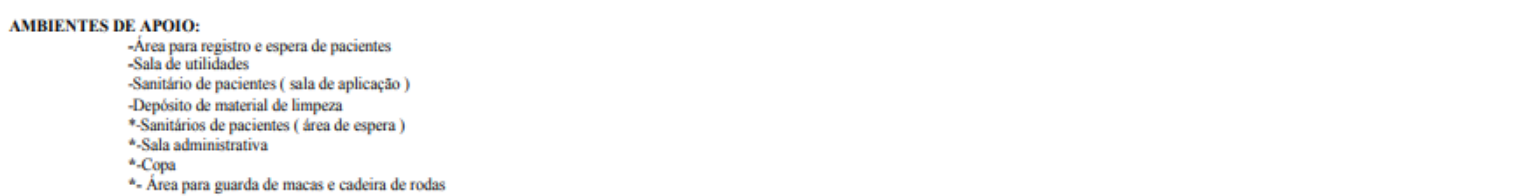 } \\
\hline $\begin{array}{ll}\text { Obs:: } & \text { 'Pode } \\
& { }^{2} \text { Vide }\end{array}$ & $\begin{array}{l}\text { alizado nos quartos ou enfermarias da intemaçăa. } \\
\text { de funcional farmsicia. }\end{array}$ & & & \\
\hline
\end{tabular}

Fig. 8: Estrutura física da unidade funcional. Fonte: Cedida pela instituição. 


\section{RECIMA21 - REVISTA CIENTÍFICA MULTIDISCIPLINAR}

DIAGNÓSTICO SITUACIONAL DE UMA CLíNICA DE TRATAMENTO ONCOLÓGICO DE BELO HORIZONTE Alice Edwirges de Castro Andrade, Daniel dos Santos Fernandes, Aline Corrêa de Almeida, Eliada Neves de Queiroz Abreu, Nayara Pereira Mota, Nicoly Cirino de Rezende, Tailane de Jesus Santos

Em conformidade com a RCD 50/2002, considera-se a RDC 220 de 21 de setembro de 2004, que dispõe sobre as condições para a promoção, proteção e recuperação da saúde de pacientes que são expostos a terapia antineoplásica e os riscos a que são expostos e descreve a saúde como direito fundamental do ser humano.

Segundo a RDC 220/2004, as unidades para terapias neoplásicas devem conter Cabine de Segurança Biológica com equipamento de proteção coletiva, insuflamento e exaustão completa de ar para proteção do produto, das pessoas e do ambiente. O Serviço de Terapia Antineoplásica (STA) deve contar com Alvará Sanitário atualizado, expedido pelo órgão sanitário competente, conforme estabelecido na Lei Federal ํㅜ6437, de 20/08/77; farmácia para preparação dos medicamentos e infraestrutura física dentro dos requisitos contidos na RDC/ANVISA № 50, de 21/02/2002.

Observando a estrutura física institucional foram encontradas evidências diferentes do que descreve a RDC 50/2002. A distância das poltronas na clínica é de $1,50 \mathrm{~m}^{2}$ a 1,70 $\mathrm{m}^{2}$ e a distância dos leitos é 2,50 $\mathrm{m}^{2}$. A RDC 50/2002 define que as poltronas nas EAS de diagnóstico e terapia voltadas para atendimentos quimioterápicos devem manter uma distância mínima de $5,0 \mathrm{~m}^{2}$ por poltrona e 7,0 $\mathrm{m}^{2}$ de distância por leito. Concordando com a RDC 50/2002, a RDC 220/2004 reforça a distância das poltronas em sala exclusiva para preparação de medicamentos para TA, com área mínima de 5,0 $\mathrm{m}^{2}$.

Nos dados coletados também existe um posto de enfermagem para cinco camas e oito poltronas; e outro posto de enfermagem com 4 poltronas presentes no $2^{\circ}$ andar. Também foi observado um posto de enfermagem para 9 camas no $4^{\circ}$ andar. A RDC 50/2002 preconiza a existência de um posto de enfermagem a cada 12 leitos/poltronas.

\section{AVALIAÇÃO DOS RECURSOS HUMANOS}

É a etapa inicial do provimento de pessoal, que tem como finalidade a previsão da quantidade de funcionários por categoria, capaz de suprir as necessidades de assistência de enfermagem, direta ou indiretamente prestada ao cliente. Compete ao enfermeiro estabelecer o quadro de profissionais necessário para a prestação da assistência de enfermagem (COREN, 2010).

Tendo como base a conceituação dessa etapa, é possível comparar o dimensionamento de pessoal do ambulatório da clínica com a proposta do Conselho Federal de Enfermagem (COFEN).

Veja o dimensionamento por sítio funcional da instituição 


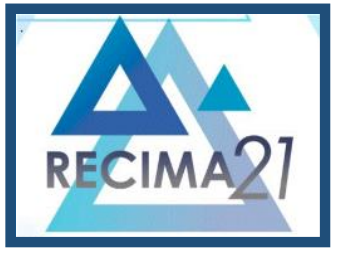

\section{RECIMA21 - REVISTA CIENTÍFICA MULTIDISCIPLINAR}

DIAGNÓSTICO SITUACIONAL DE UMA CLíNICA DE TRATAMENTO ONCOLÓGICO DE BELO HORIZONTE Alice Edwirges de Castro Andrade, Daniel dos Santos Fernandes, Aline Corrêa de Almeida, Eliada Neves de Queiroz Abreu, Nayara Pereira Mota, Nicoly Cirino de Rezende, Tailane de Jesus Santos

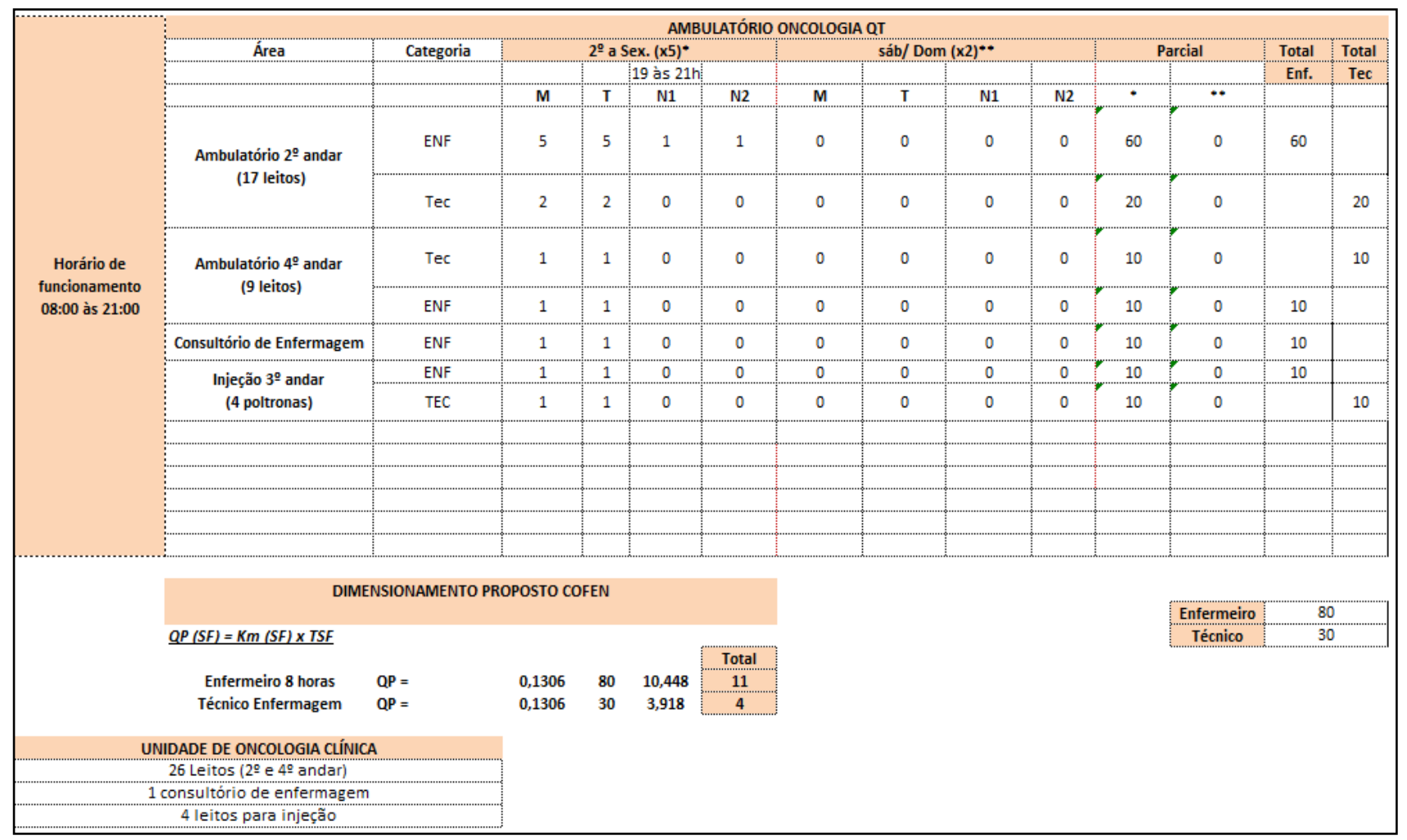

Fig. 9: Tabela de dimensionamento de pessoal da clínica conforme Cofen. Fonte: Cedida pela instituição.

Para realizar o cálculo de dimensionamento de pessoal a clínica utiliza essa planilha, modelo padrão do Cofen, preenchendo as particularidades do serviço que é classificado como Unidade Assistencial Especial (UAE). Para o cálculo de dimensionamento por sítio funcional, a clínica utiliza a fórmula disposta na Resolução 543/2017: QP(SF) = KM X TSF, onde QP é igual a quantidade de pessoal, KM é igual a Constante de Marinho e TSF é total de sítios funcionais.

$\mathrm{Na}$ Resolução 543/2017, que dispõe sobre esse dimensionamento de enfermagem, são estabelecidos os números mínimos de profissionais de enfermagem para cada paciente, sendo eles enfermeiros, técnicos e auxiliares. Cabe ao enfermeiro o planejamento e coordenação dos serviços de assistência de enfermagem, sendo ele responsável por fazer o cálculo de dimensionamento para a instituição (pública ou privada) se adequar aos requisitos pelo Cofen. Para que o dimensionamento ocorra corretamente, é necessário um instrumento de classificação do paciente, que deverá ser utilizado pelo enfermeiro responsável, observando o tempo médio de cuidado para cada paciente de acordo com seu grau de dependência. 


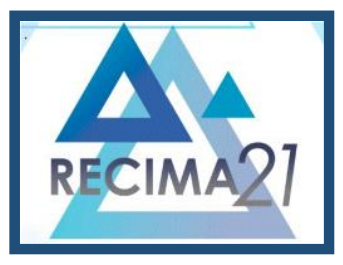

\section{RECIMA21 - REVISTA CIENTÍFICA MULTIDISCIPLINAR}

DIAGNÓSTICO SITUACIONAL DE UMA CLÍNICA DE TRATAMENTO ONCOLÓGICO DE BELO HORIZONTE Alice Edwirges de Castro Andrade, Daniel dos Santos Fernandes, Aline Corrêa de Almeida, Eliada Neves de Queiroz Abreu, Nayara Pereira Mota, Nicoly Cirino de Rezende, Tailane de Jesus Santos

A figura 10 é a representação gráfica da distribuição das áreas operacionais com dias da semana, turnos e categorias profissionais. Esse é o modelo disposto pelo Cofen e utilizado pela clínica papa o dimensionamento.

\begin{tabular}{|c|c|c|c|c|c|c|c|c|c|c|c|c|c|}
\hline \multirow{3}{*}{$\begin{array}{l}\text { Área Operacional } \\
\text { (Local da Atividade) }\end{array}$} & \multirow{3}{*}{$\begin{array}{l}\text { Categoria } \\
\text { Profissional } \\
\end{array}$} & \multicolumn{7}{|c|}{ ESPELHO SEMANAL PADRÃO } & & & \multirow{3}{*}{\begin{tabular}{|c} 
Subtotal de SF \\
$\times 2$ \\
\end{tabular}} & \multirow{3}{*}{$\begin{array}{c}\text { Total de SF } \\
\text { NS } \\
\end{array}$} & \multirow{3}{*}{\begin{tabular}{|c} 
Total de S \\
NM
\end{tabular}} \\
\hline & & \multicolumn{4}{|c|}{$2 \mathrm{a}$ a $6 \mathrm{a}$ Feira } & \multirow{2}{*}{\begin{tabular}{|c} 
Subtotal de SF \\
$\times 5$ \\
\end{tabular}} & \multicolumn{4}{|c|}{ Sábado e Domingo } & & & \\
\hline & & $M$ & $\mathrm{~T}$ & N1 & N2 & & $M$ & $\mathrm{~T}$ & N1 & $\mathrm{N} 2$ & & & \\
\hline & NS & & & & & & & & & & & & \\
\hline & NM & & & & & & & & & & & & \\
\hline & NS & & & & & & & & & & & & \\
\hline & NM & & & & & & & & & & & & \\
\hline & MS & & & & & & & & & & & & \\
\hline & NM & & & & & & & & & & & & \\
\hline
\end{tabular}

Fig. 10: Tabela para cálculo de dimensionamento de pessoal proposto pelo Cofen. Fonte: COREN-MG.

Conforme observado na tabela de dimensionamento de pessoal da clínica, o cálculo disposto na Resolução 543/2017 é aplicado, tendo como resultado a necessidade de 11 enfermeiros para 4 técnicos de enfermagem. Ao analisar a disposição de enfermeiros e técnicos da instituição, é possível perceber um número de enfermeiros acima do exigido, sendo 11 assistenciais e 2 supervisores. Já a quantidade de técnicos de enfermagem é inferior a quantidade exigida pelo Cofen, sendo apenas 2. Porém, essa disposição é compensatória, já que o serviço exige mais enfermeiros do que técnicos devido à complexidade dos pacientes e dos procedimentos privativos ao enfermeiro.

\section{AVALIAÇÃO DOS RECURSOS MATERIAIS}

A gestão de materiais proporciona a dimensão organizativa de uma instituição, influencia diretamente no funcionamento adequado, no desempenho geral da organização, nos serviços de saúde, impactando na promoção, proteção e recuperação da saúde das pessoas. (COREN, 2020)

Segundo o Conselho Regional de Enfermagem (COREN, 2020), o gerenciamento desta atividade tem como objetivos o fornecimento de recursos necessários ao trabalho com qualidade e quantidades adequadas, baixos custos e tempo hábil.

É perceptível o empenho da instituição em prover ferramentas de planejamentos e gestão para manter disponível os adequados recursos materiais. $O$ dimensionamento adequado deste recurso contribui significativamente com a segurança e qualidade assistencial.

A fig. 11 demonstra resumidamente como estão disponibilizados equipamentos, insumos, materiais, medicamentos e mobiliário na clínica. 


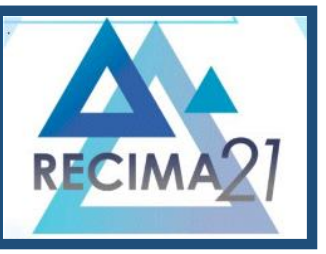

\section{RECIMA21 - REVISTA CIENTÍFICA MULTIDISCIPLINAR}

DIAGNÓSTICO SITUACIONAL DE UMA CLÍNICA DE TRATAMENTO ONCOLÓGICO DE BELO HORIZONTE Alice Edwirges de Castro Andrade, Daniel dos Santos Fernandes, Aline Corrêa de Almeida, Eliada Neves de Queiroz Abreu, Nayara Pereira Mota, Nicoly Cirino de Rezende, Tailane de Jesus Santos

\begin{tabular}{|c|c|c|c|c|c|}
\hline \multicolumn{6}{|c|}{ Recursos materiais e equipamentos } \\
\hline \multirow{4}{*}{$\begin{array}{l}\text { Materiais } \\
\text { consumiveis }\end{array}$} & \begin{tabular}{|l|} 
Materiais $\mathrm{e}$ \\
equipamentos \\
\end{tabular} & Armaxenamento & Liberaçăo & Controle & Acesso \\
\hline & $\begin{array}{l}\text { Algodja } \\
\text { Alcool } \\
\text { Riohex } 0.5 \% \\
\text { Pohehex } 2 \% \\
\text { Luvas estiereis } \\
\text { Luvas de } \\
\text { procedimentos } \\
\text { Mascara } \\
\text { Gorro/touca } \\
\text { Capote } \\
\text { Garrote } \\
\text { Micropore } \\
\text { Transporte }\end{array}$ & $\begin{array}{l}\text { Armazzenados no } \\
\text { posto de } \\
\text { enfermagem em } \\
\text { locais } \\
\text { especificos } \\
\text { (armários } \\
\text { identificados) }\end{array}$ & $\begin{array}{l}\text { Liberado pela } \\
\text { farmácia após } \\
\text { preenchimento } \\
\text { de formulario } \\
\text { específico com } \\
\text { limites de } \\
\text { quansiades por } \\
\text { material }\end{array}$ & $\begin{array}{l}\text { Requisicão } \\
\text { diária mediante } \\
\text { quantidade } \\
\text { paddronizada } \\
\text { (check list) } \\
\text { para cadia setor }\end{array}$ & $\begin{array}{l}\text { Disponivel no posto de } \\
\text { enfermagem mediante o } \\
\text { término do produto } \\
\text { anterior }\end{array}$ \\
\hline & $\begin{array}{l}\text { Seringas } \\
\text { Agulhas } \\
\text { Equipo } \\
\text { Edensor } \\
\text { Scalp e jelco } \\
\text { Tree Way } \\
\text { Gases } \\
\text { Medicamentos }\end{array}$ & $\begin{array}{l}\text { Farmácia } \\
\text { Enfermagem } \\
\text { (maleta de } \\
\text { emergếncia) }\end{array}$ & $\begin{array}{l}\text { Liberação e } \\
\text { reposicão pela } \\
\text { farmácia, } \\
\text { mediante } \\
\text { cobranca de } \\
\text { materiais na } \\
\text { conta do } \\
\text { paciente }\end{array}$ & $\begin{array}{l}\text { Controle e } \\
\text { rastreabilidade } \\
\text { via sistema. } \\
\text { monitorando } \\
\text { lote, validade e } \\
\text { obdigo de barra } \\
\text { (maleta ou } \\
\text { dispensac.so } \\
\text { direta pela } \\
\text { farmácia) }\end{array}$ & $\begin{array}{l}\text { Disponível no posto de } \\
\text { enfermagem, reposiç̃o } \\
\text { mediante cobrança em } \\
\text { conta (paciente)- } \\
\text { requisiçio aulomática }\end{array}$ \\
\hline & $\begin{array}{l}\text { Maleta de } \\
\text { emergéncia } 2^{\circ} \mathrm{e} \\
4^{\circ} \text { andar }\end{array}$ & $\begin{array}{l}\text { Armazenados no } \\
\text { posto de } \\
\text { enfermagem } \\
2^{\circ} \cdot 4^{*} \text { andar }\end{array}$ & $\begin{array}{l}\text { Liberação e } \\
\text { reposichäo pela } \\
\text { tarmacia, } \\
\text { mediante } \\
\text { cobranca de } \\
\text { materiais na } \\
\text { conta do } \\
\text { paciente }\end{array}$ & $\begin{array}{l}\text { Controle e } \\
\text { rastreabilidade } \\
\text { va sistema. } \\
\text { monitorando } \\
\text { lote, validade e } \\
\text { ódigo de barra }\end{array}$ & $\begin{array}{l}\text { Disponivel no posto de } \\
\text { enfermagem realizando a } \\
\text { cobranca mediante ao uso }\end{array}$ \\
\hline \multirow[b]{2}{*}{$\begin{array}{l}\text { Materiais } \\
\text { permanentes }\end{array}$} & $\begin{array}{l}\text { Materiais } \\
\text { reprocessados } \\
\text { Umidificador } \\
\text { Macronebulizador } \\
\text { Micronebulizador } \\
\text { BMM } \\
\text { Máscara } \\
\text { reservatónia } \\
\text { Látex } \\
\text { Cånula de guedel }\end{array}$ & $\begin{array}{l}\text { Armazenados no } \\
\text { posto de } \\
\text { enfermagem em } \\
\text { locais } \\
\text { especificos } \\
\text { (apmários } \\
\text { identificados) e } \\
\text { carrinho de } \\
\text { emergéncia }\end{array}$ & $\begin{array}{l}\text { Inventário dos } \\
\text { maleriais de } \\
\text { CME após } \\
\text { reprocessamento } \\
\text { (terceirizado } \\
\text { ETO) }\end{array}$ & $\begin{array}{l}\text { Hens } \\
\text { disponiveis no } \\
\text { estoque } \\
\text { (controle CME) } \\
\text { ambulatórios e } \\
\text { carrinho de } \\
\text { emergência. } \\
\text { Requisicảo } \\
\text { automitica. } \\
\text { mediantio ao } \\
\text { lancamento do } \\
\text { iterm com } \\
\text { ódigo de } \\
\text { barras na conta } \\
\text { do paciente }\end{array}$ & $\begin{array}{l}\text { Disponivel no posto de } \\
\text { enfermagem realizando a } \\
\text { cobranca mediante ao uso }\end{array}$ \\
\hline & $\begin{array}{l}\text { Palm } \\
\text { Bandejas } \\
\text { Oximetro } \\
\text { Aspirador } \\
\text { Desfiorilador } \\
\text { Cardioversor } \\
\text { Laringoscóplio } \\
\text { Termómetro } \\
\text { Aparelho de PA } \\
\text { Comocaato- } \\
\text { Bomba de infusào } \\
\text { Aparelho capilar } \\
\text { hipotérmico } \\
\text { Termostatos }\end{array}$ & $\begin{array}{l}\text { Armazenades no } \\
\text { posto de } \\
\text { enfermagem em } \\
\text { locais } \\
\text { especificos } \\
\text { (armáicos } \\
\text { identificados) e } \\
\text { carrinho de } \\
\text { emergéncia }\end{array}$ & $\begin{array}{l}\text { Itens } \\
\text { permanentes: } \\
\text { compra } \\
\text { provisionada } \\
\text { conforme } \\
\text { necessidade. } \\
\text { Equipamentos } \\
\text { disponibilizados } \\
\text { para yso } \\
\text { contorme } \\
\text { legislaçäo e } \\
\text { necessidade de } \\
\text { cada setor }\end{array}$ & $\begin{array}{l}\text { Controle đos } \\
\text { bens } \\
\text { permanentes: } \\
\text { equipamentos } \\
\text { com placa de } \\
\text { património, } \\
\text { controle anual } \\
\text { de } \\
\text { greventivas } \\
\text { manutenção } \\
\text { biomédica đe } \\
\text { todos os } \\
\text { equipamentos } \\
\text { e } \\
\text { manutenção } \\
\text { corretiva } \\
\text { conforme } \\
\text { necessidade }\end{array}$ & $\begin{array}{l}\text { Disponivel no posto de } \\
\text { enfermagem e carrinho de } \\
\text { emergéncia }\end{array}$ \\
\hline Imobiliários & $\begin{array}{l}\text { Poltronas } \\
\text { Camas } \\
\text { Armilios } \\
\text { Suporte para } \\
\text { Soro } \\
\text { Biombo } \\
\text { Maca } \\
\text { Balança }\end{array}$ & $\begin{array}{l}\text { Disponivel nos } \\
\text { postos de } \\
\text { enfermagem e } \\
\text { sala de utilidade }\end{array}$ & $\begin{array}{l}\text { Itens } \\
\text { imobiliários: } \\
\text { compra } \\
\text { provisionada } \\
\text { conforme } \\
\text { necessidade. } \\
\text { Equipamentos } \\
\text { disponibilizados } \\
\text { para uso } \\
\text { conforme } \\
\text { legislacão e } \\
\text { necessidade de } \\
\text { cada setor }\end{array}$ & $\begin{array}{l}\text { Planiliha com } \\
\text { identificador } \\
\text { patrimonial e } \\
\text { código de } \\
\text { barra }\end{array}$ & $\begin{array}{c}\text { Disponivel nos postos de } \\
\text { enfermagem }\end{array}$ \\
\hline $\begin{array}{c}\text { Materiais } \\
\text { Administrativos }\end{array}$ & $\begin{array}{l}\text { Folhas A4 } \\
\text { Computadores } \\
\text { Cadeiras } \\
\text { Apoio para os } \\
\text { pes } \\
\text { Impressoras } \\
\text { Canetas e pincéis } \\
\text { Artilhas de } \\
\text { orientaçōes }\end{array}$ & $\begin{array}{l}\text { Armazenados no } \\
\text { posto de } \\
\text { enfermagem em } \\
\text { locais } \\
\text { especifices } \\
\text { (armínios } \\
\text { identificados) }\end{array}$ & $\begin{array}{l}\text { Suprimentos } \\
\text { após a realcaçăo } \\
\text { de requisiç̄o de } \\
\text { materiais }\end{array}$ & $\begin{array}{l}\text { Requisicão } \\
\text { semanal via } \\
\text { sistema com } \\
\text { limiles de } \\
\text { quantidade }\end{array}$ & $\begin{array}{c}\text { Disponivel nos postos de } \\
\text { enfermagem }\end{array}$ \\
\hline
\end{tabular}




\section{RECIMA21 - REVISTA CIENTÍFICA MULTIDISCIPLINAR}

DIAGNÓSTICO SITUACIONAL DE UMA CLÍNICA DE TRATAMENTO ONCOLÓGICO DE BELO HORIZONTE Alice Edwirges de Castro Andrade, Daniel dos Santos Fernandes, Aline Corrêa de Almeida, Eliada Neves de Queiroz Abreu, Nayara Pereira Mota, Nicoly Cirino de Rezende, Tailane de Jesus Santos

As manutenções são ações destinadas no auxílio de conservação e recuperações de materiais e equipamentos. A equipe de manutenção apoiará toda a instituição mantendo a funcionalidade e garantindo a qualidade dos produtos existentes, podendo ser de forma preventiva ou corretiva (ABNT/1994, p. 6).

A manutenção corretiva é efetuada após a ocorrência de uma pane destinada a recolocar um item em condições de executar uma função requerida. Já a manutenção preventiva é efetuada em intervalos predeterminados, ou de acordo com critérios prescritos, destinada a reduzir a probabilidade de falha ou a degradação do funcionamento de um item (ABNT, NBR 5462/1994, p. 7).

Nos quadros abaixo são evidenciados os recursos dispostos pela instituição para manter o controle, a qualidade e a quantidade adequada dos equipamentos para um bom funcionamento das atividades assistenciais e sistêmicas.

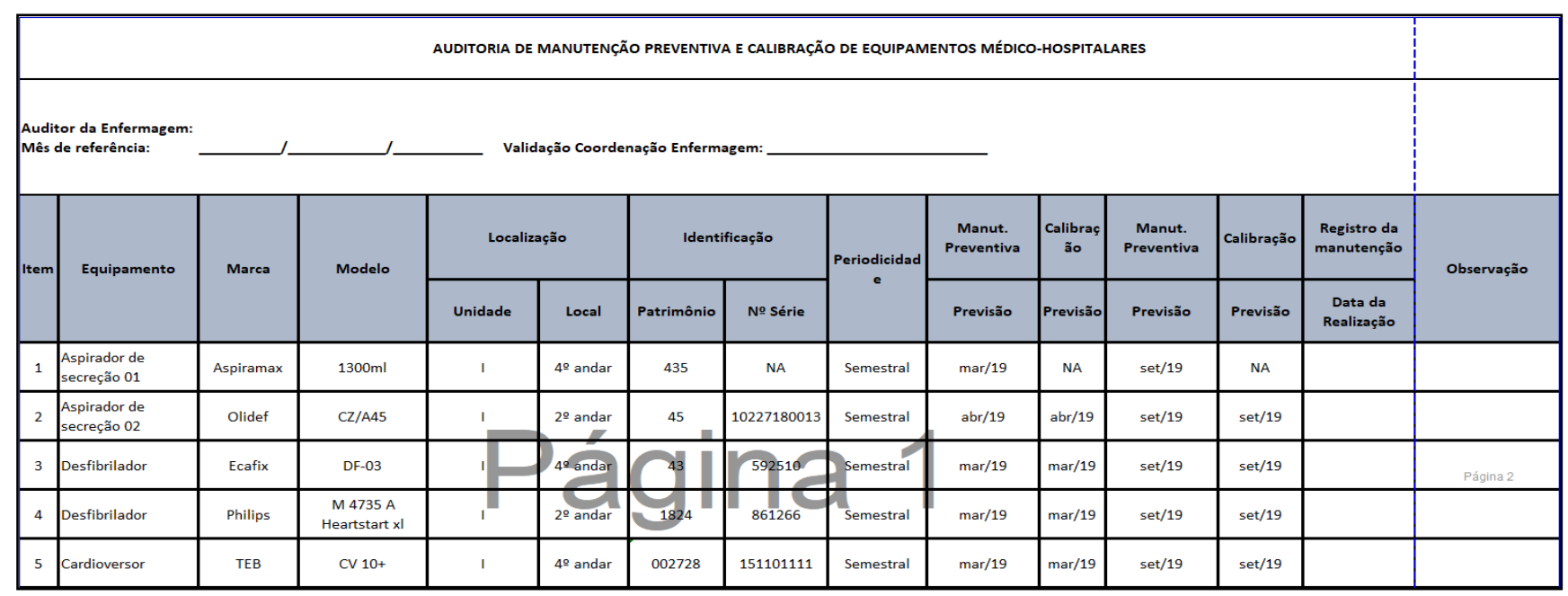

Fig. 12: Auditoria de Manutenção preventiva. Fonte: Cedida pela instituição.

\begin{tabular}{|c|c|c|c|c|c|c|c|c|c|c|}
\hline \multirow{2}{*}{ Item } & \multirow{2}{*}{ Equipamento } & \multirow{2}{*}{ Marca } & \multirow{2}{*}{ Modelo } & \multicolumn{2}{|c|}{ Localização } & \multicolumn{2}{|c|}{ Identificação } & \multirow{2}{*}{ Periodicidade } & \multirow{2}{*}{$\begin{array}{c}\text { Inspeção do IPEM } \\
\text { Previsão }\end{array}$} & \multirow{2}{*}{$\frac{\text { Calibração }}{\text { Previsão }}$} \\
\hline & & & & Unidade & Local & Patrimônio & № Série & & & \\
\hline 1 & Balança & Balmak & Antopométrica & I & 5 andar & 565 & 9979 & Anual & $\mathrm{fev} / 20$ & set $/ 20$ \\
\hline 2 & Balança & Filizola & Antopométrica & $\mathrm{I}$ & \begin{tabular}{|l|} 
Cons. 5 \\
\end{tabular} & 824 & 62998 & Anual & $\mathrm{fev} / 20$ & set $/ 20$ \\
\hline 3 & Balança & Arja & Antopométrica & $\mathrm{I}$ & Cons. 1 & 904 & 25823 & Anual & $\mathrm{fev} / 20$ & set $/ 20$ \\
\hline 4 & Balança & Welmy & Antopométrica & 1 & Cons. 12 & 986 & 59251 & Anual & $\mathrm{fev} / 20$ & set $/ 20$ \\
\hline 5 & Balança Eletronica & Welmy & W 300 & 1 & HIG & 2160 & 6867 & Anual & $\mathrm{fev} / 20$ & set $/ 20$ \\
\hline 6 & Balança & Welmy & Antropometrica & 1 & \begin{tabular}{|l|} 
Cons. 6 \\
\end{tabular} & 2343 & 126174 & Anual & $\mathrm{fev} / 20$ & set $/ 20$ \\
\hline 7 & Balança & Welmy & $110 \mathrm{CH}$ & I & 60 andar & 2775 & 136862 & Anual & $\mathrm{fev} / 20$ & set $/ 20$ \\
\hline 8 & Balança & Welmy & $110 \mathrm{CH}$ & 1 & \begin{tabular}{|l|} 
Cons. 9 \\
\end{tabular} & 2776 & 136434 & Anual & $\mathrm{fev} / 20$ & set $/ 20$ \\
\hline 9 & Balança & Welmy & $110 \mathrm{CH}$ & I & Cons. 4 & 2777 & 137061 & Anual & $\mathrm{fev} / 20$ & set $/ 20$ \\
\hline 10 & Balança & Welmy & $110 \mathrm{CH}$ & 1 & Cons. 2 & 3058 & 140668 & Anual & $\mathrm{fev} / 20$ & set/20 \\
\hline 11 & Balança & Welmy & $110 \mathrm{CH}$ & I & Cons. 3 & 3059 & 140666 & Anual & $\mathrm{fev} / 20$ & set $/ 20$ \\
\hline 12 & Balança & Welmy & $110 \mathrm{CH}$ & 1 & Cons. 7 & 3060 & 140673 & Anual & $\mathrm{fev} / 20$ & set $/ 20$ \\
\hline 13 & Balança & Welmy & $110 \mathrm{CH}$ & I & Cons. 8 & 3061 & 140669 & Anual & $\mathrm{fev} / 20$ & set $/ 20$ \\
\hline 14 & Balança & Welmy & $110 \mathrm{CH}$ & 1 & Cons. 11 & 3062 & 140667 & Anual & $\mathrm{fev} / 20$ & set $/ 20$ \\
\hline 15 & Balança & Welmy & $110 \mathrm{CH}$ & $\mathrm{I}$ & Cons. 13 & 3063 & 140671 & Anual & $\mathrm{fev} / 20$ & set $/ 20$ \\
\hline 16 & Balança & Welmy & $110 \mathrm{CH}$ & II & Cons. 5 & 3021 & 143287 & Anual & $\mathrm{fev} / 20$ & set $/ 20$ \\
\hline 17 & Balança & Welmy & $110 \mathrm{CH}$ & II & Cons. 1 & 3248 & 2772 & Anual & $\mathrm{fev} / 20$ & set $/ 20$ \\
\hline 18 & Valvula reguladora & \begin{tabular}{|l|} 
White Martins \\
\end{tabular} & NA & 1 & \begin{tabular}{|l|} 
reserva \\
\end{tabular} & 2277 & NA & Anual & NA & nov $/ 20$ \\
\hline 19 & Valvula reguladora & Protec & $\mathrm{NA}$ & 1 & G - 49 andar & 2278 & NA & Anual & NA & nov $/ 20$ \\
\hline 20 & Valvula reguladora & White Martins & NA & 1 & $6 \mathrm{~m}^{3}$ & 2280 & NA & Anual & NA & nov $/ 20$ \\
\hline 21 & Valvula reguladora & White Martins & $\mathrm{NA}$ & 1 & G-29 andar & 2922 & NA & Anual & NA & nov $/ 20$ \\
\hline 22 & Valvula reguladora & Protec & NA & $\mathrm{I}$ & white med & 999014 & NA & Anual & NA & nov $/ 20$ \\
\hline
\end{tabular}

Fig. 13: Cronograma de manutenção preventiva. Fonte: Cedida pela instituição. 


\section{RECIMA21 - REVISTA CIENTÍFICA MULTIDISCIPLINAR}

A enfermagem é a maior usuária destes recursos, sendo relevante a contribuição na contenção de gastos, educação continuada, adequação ao espaço físico e aspectos quantiqualitativo dos recursos (COREN, 2020).

\begin{tabular}{|c|c|}
\hline \multicolumn{2}{|c|}{ Maleta de emergência } \\
\hline Descrição & Quantidade \\
\hline ABD 20ml (ampola 20ml) & 5 \\
\hline Agulha desc. $40 / 12 \mathrm{c} /$ dispositivo & 10 \\
\hline Atropina $0,5 \mathrm{mg} / \mathrm{ml}$ inj. (ampola $1 \mathrm{ml}$ ) & 2 \\
\hline Brometo de N-Butilescopolamina 20mg/ml (amp. 1ml) & 2 \\
\hline Captopril 25mg & 2 \\
\hline Cateter intra venoso $n^{\circ} 22$ & 10 \\
\hline Cateter intra venoso $n^{\circ} 20$ & 1 \\
\hline Cateter intra venoso $n^{\circ} 24$ & 10 \\
\hline Cateter p/ oxigênio tipo óculos & 2 \\
\hline Cloreto de Sódio 0,9\% ampola c/ $10 \mathrm{ml}$ & 5 \\
\hline Cloreto de Sódio 0,9\% frasco 100ml & 2 \\
\hline Cloreto de Sódio 0,9\% $250 \mathrm{ml}$ & 2 \\
\hline Cloreto de Sódio 0,9\% frasco 500ml & 2 \\
\hline Dipirona $2 \mathrm{ml}$ amp. 500mg/ml & 3 \\
\hline Dispositivo de infusão múltiplas 3 vias & 1 \\
\hline Dispositivo de infusão periférica c/ asas $n^{\circ} 21$ & 2 \\
\hline Dramin B6 DL inj. Ampola 10ml & 3 \\
\hline Equipo p/ Bl & 2 \\
\hline Equipo simples & 3 \\
\hline Extensão p/ perfusão & 2 \\
\hline Glicose $5 \% 100 \mathrm{ml}$ sistema fechado & 2 \\
\hline Glicose $5 \% 500 \mathrm{ml}$ sistema fechado & 1 \\
\hline Hidrocortisona 100mg inj. (frasco-ampola pó) & 8 \\
\hline Isordil 05 mg cp SL & 2 \\
\hline Maleato de Dexclorfeniramina $2 \mathrm{mg} \mathrm{cp}$ & 6 \\
\hline Metoclopramida $5 \mathrm{mg}$ ampola $2 \mathrm{ml}$ & 5 \\
\hline Nifedipina R 10mg cp & 2 \\
\hline Seringa desc. $20 \mathrm{ml}$ Luerlock & 5 \\
\hline Seringa desc. 10ml Luerlock & 10 \\
\hline Seringa desc. 05ml Luerlock & 5 \\
\hline Seringa $10 \mathrm{ml}$ Sol. Salina $0,9 \%$ & 3 \\
\hline Tira teste $\mathrm{p} /$ glicose & 5 \\
\hline
\end{tabular}

Fig. 14: Maleta de urgência. Fonte: Criação dos autores. 


\section{RECIMA21 - REVISTA CIENTÍFICA MULTIDISCIPLINAR}

DIAGNÓSTICO SITUACIONAL DE UMA CLÍNICA DE TRATAMENTO ONCOLÓGICO DE BELO HORIZONTE Alice Edwirges de Castro Andrade, Daniel dos Santos Fernandes, Aline Corrêa de Almeida, Eliada Neves de Queiroz Abreu, Nayara Pereira Mota, Nicoly Cirino de Rezende, Tailane de Jesus Santos

Observa-se o alinhamento entre os profissionais multidisciplinares. Antes de realizar a dispensação dos materiais e medicamentos para a enfermagem, nota-se o cuidado dos profissionais da farmácia mediante suas atividades diárias, mantendo a padronização para o recebimento, controle, identificação e armazenamento dos materiais e medicamentos MAT/MED (RDC45).

Conforme descrito abaixo, o controle de rastreabilidade das maletas de emergência encontrados no posto de enfermagem do segundo e quarto andar, são realizados pelos farmacêuticos através de cobrança via aquisição automática com identificação MAT/MED (contendo lote, código de barra, validade e nome do material e medicamento).

\begin{tabular}{|c|c|c|c|}
\hline Material Descrição material & & Qt estoqu & \\
\hline Seq lote Descrição lote Validade & Barras & Qt estoqu & \\
\hline Maleta emergência 2 andar & & & \\
\hline 45 Agua Bidestilada $20 \mathrm{ml}$ (am & $\mathrm{c} / 20 \mathrm{~mL})$ & 4,000 & \\
\hline 81643 AVV2 & $31 / 01 / 2022$ & 00000816434 & 4,0000 \\
\hline 50293 Agulha descartável $40 \times 12$ & lispositivo de & 9,000 & \\
\hline 829820121011 & $30 / 04 / 2025$ & 00000829820 & 2,0000 \\
\hline 820959352348 & $31 / 12 / 2024$ & 00000820954 & 7,0000 \\
\hline 561 Atropina ampola $0,5 \mathrm{mg} / \mathrm{mL}$ & & 2,000 & \\
\hline 8491520060946 & $30 / 06 / 2022$ & 00000849154 & 2,0000 \\
\hline 212 Butilescopolamina $20 \mathrm{mg} / \mathrm{mL}$ & npola $1 \mathrm{~mL}$ & 2,000 & \\
\hline 83680 B68172 & $30 / 09 / 2024$ & 00000836800 & 1,0000 \\
\hline $85838 \quad \mathrm{~B} 68173$ & $30 / 09 / 2024$ & 00000858382 & 1,0000 \\
\hline 849 Captopril 25mg comp & & 2,000 & \\
\hline $82162 \quad 1 \mathrm{~L} 8504$ & $30 / 11 / 2021$ & 00000821624 & 2,0000 \\
\hline 1062 Catéter p/ oxigênio tipo ócu & & 1,000 & \\
\hline
\end{tabular}

Fig. 15: Controle de Rastreabilidade da Maleta de Emergência. Fonte: Cedida pela instituição.

A reposição destas maletas será realizada mediante a cobrança da enfermagem na conta do paciente, via sistema, a partir do código de barras contido no material ou medicação. A equipe de enfermagem utilizará os MAT/MED contidos na maleta de emergência, caso haja necessidade. Para os materiais serão exigidos a justificativa de materiais extras e para medicamentos será necessário a apresentação da prescrição médica; salvo em caso de urgência e emergência (COFEN, 2015).

É de responsabilidade da enfermagem a cobrança imediata do MAT/MED para que não haja perda de rastreabilidade. A conferência dos itens utilizados será realizada diariamente pela enfermagem.

Os medicamentos dispensados pela farmácia para infusão parenteral deverão ser armazenados pela equipe de enfermagem em locais que mantenham a segurança e que descartem a possibilidade de queda, ruptura e contaminação do ambiente. Em caso de acidentes (derramamento), os colaboradores utilizarão kits (maleta de derramamento) disponibilizados na farmácia ( $2^{\circ}$ andar), no posto de enfermagem $4^{\circ}$ andar e suprimentos (unidade III).

O local de armazenamento deverá ser tal que evite a queda e ruptura dos recipientes, não esquecendo que tais medicamentos podem necessitar de condições especiais de armazenamento (ANVISA, 2003). 


\section{RECIMA21 - REVISTA CIENTÍFICA MULTIDISCIPLINAR}

INSTRUMENTOS GERENCIAIS

DIAGNÓSTICO SITUACIONAL DE UMA CLÍNICA DE TRATAMENTO ONCOLÓGICO DE BELO HORIZONTE Alice Edwirges de Castro Andrade, Daniel dos Santos Fernandes, Aline Corrêa de Almeida, Eliada Neves de Queiroz Abreu, Nayara Pereira Mota, Nicoly Cirino de Rezende, Tailane de Jesus Santos

Os instrumentos gerenciais são ferramentas que embasam a organização e funcionamento dos serviços de enfermagem. Esses recursos servem de apoio à função gerencial do enfermeiro, visando torna-la assertiva e sistematizada (COREN-MG, 2020).

Os instrumentos gerenciais servem de apoio à tomada de decisão do enfermeiro, dão apoio e suporte ao planejamento, visando alcançar um objetivo específico. Todas essas características e funções de cada instrumento gerencial apresentado fazem parte da organização da clínica em estudo.

Foi evidenciado que o serviço faz uso de vários instrumentos gerenciais para tomada de decisão. O gerenciamento do processo de trabalho da enfermagem tem como principal objetivo a organização da assistência, por isso, para um bom funcionamento, a instituição deve fazer uso de diversos recursos de gerenciamento.

Segundo Silva e Fontoura (2019), no processo de gerenciamento da enfermagem, os objetos de trabalho do enfermeiro são a organização do trabalho e dos recursos humanos. Esses recursos torna a instituição capaz de administrar a assistência de enfermagem, conhecer problemas mais comuns, levantar as necessidades dos clientes, gerenciar conflitos e capacitar a equipe.

A instituição dispõe de diversos recursos gerenciais que a habilita a tornar a assistência assertiva, resolutiva e organizada. Nesse sentido, foram identificados e analisados alguns instrumentos gerenciais disponíveis no serviço:

\section{Procedimento Sistêmico (PRS)}

É um documento que registra procedimentos que envolvem mais de um setor ou serviço, representando uma rotina de trabalho que deve ser seguida por toda a instituição. Todos os setores têm acesso ao PRS.

\begin{tabular}{|c|c|c|c|}
\hline Código & Revisão & Documento & Tipo doc \\
\hline PिRS-ENF-0 $\overline{01}$ & 12 & \multicolumn{2}{|c|}{ Referência e Transferência do paciente para a Unidade Hospit PRS } \\
\hline PRS-ENF-002 & 10 & Controle do Caminho de Emergência e maletas de apoio & PRS \\
\hline PRS-ENF-004 & 06 & \multicolumn{2}{|l|}{ Atendimento à intercorrência com o paciente durante a adminis PRS } \\
\hline PRS-ENF-006 & 04 & Requisição e devolução de Mat/Med no ambulatório (00) & PRS \\
\hline PRS-ENF-007 & 02 & \multicolumn{2}{|l|}{ Cobrança de Taxas de Equipamentos, Procedimentos e Oxigêı PRS } \\
\hline PRS-ENF-008 & 02 & Gerenciamento de Riscos Assistênciais na Oncologia & PRS \\
\hline PRS-ENF-009 & & Atendimento inicial e monitoramento do paciente oncológico & PRS \\
\hline
\end{tabular}

Fig. 16: Lista de PRS. Fonte: Cedida pela instituição.

\section{Procedimento Operacional Padrão (POP)}




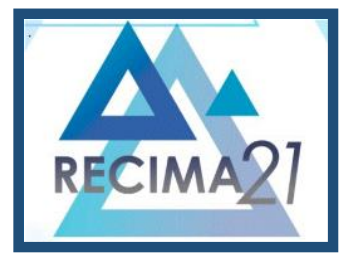

\section{RECIMA21 - REVISTA CIENTÍFICA MULTIDISCIPLINAR} DIAGNÓSTICO SITUACIONAL DE UMA CLíNICA DE TRATAMENTO ONCOLÓGICO DE BELO HORIZONTE
Alice Edwirges de Castro Andrade, Daniel dos Santos Fernandes, Aline Corrêa de Almeida, Eliada Neves de Queiroz Abreu,

É um documento claro e objetivo que visa descrever sistematicamente o passo a passo de um procedimento naquele serviço. A instituição conta as seguintes informações no documento: executores da atividade, materiais, tarefas críticas, siglas e definições e detalhamentos das atividades. A figura 17 é parte do POP utilizado pela instituição. O modelo apresentado pela instituição corresponde aos itens recomendados pelo Conselho Regional de Enfermagem de Sergipe (2017). A descrição detalhada das atividades, de forma clara e objetiva, classificação dos executores, descrição de tarefas críticas, enfim, a uniformização da assistência prestada.

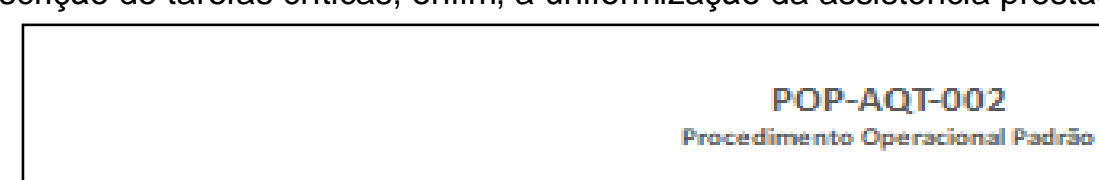

Nome: Escalas e rotinas da Equipe de Enfermagem

\section{EXECUTORES DAATMIDADE}

Enfermeiro e Técnico de Enfermagern;

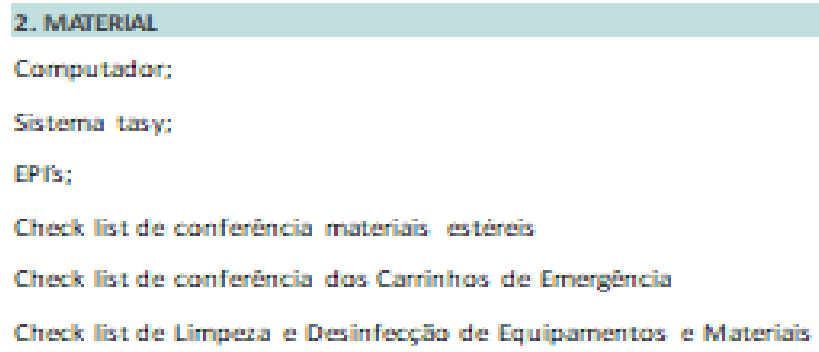

\section{TAREFA CRITICA}

Execuçāo das atividades conforme escala.

\section{SIGLAS E DEFINIÇŌES}

Escala Mensal: Dimensionamento da equipe de enfermagem em todos as ambulató rios de $2^{3}$ a 63 feira conforme legislaçāo.

Escala de tar efas: Elaborada mensalmente para plan ejamento das atividades diárias, semanais e mens ais da equipe de enfermagem.

Escalas de leito: Para dimension amento dos enfermeiros nas unid ades ambulatoriais. Atu alizad a seman al mente.

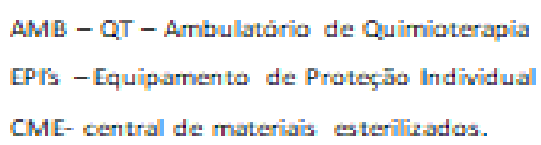

\section{DETALHAMENTO DAS ATMIDADES}

Escalas

A equipede enfermagem execut ar á as ativid ades de acordo com 03 escalas distintas ques äo disponibilizadas nos postos de enfermagem. Tais atividades seräo validadas pelo enfermeiro lider durante a jornada de trabalho.

Fig. 17: Modelo de POP. Fonte: Cedida pela instituição. 


\section{RECIMA21 - REVISTA CIENTÍFICA MULTIDISCIPLINAR}

DIAGNÓSTICO SITUACIONAL DE UMA CLÍNICA DE TRATAMENTO ONCOLÓGICO DE BELO HORIZONTE Alice Edwirges de Castro Andrade, Daniel dos Santos Fernandes, Aline Corrêa de Almeida, Eliada Neves de Queiroz Abreu,

\begin{tabular}{|c|c|c|c|c|}
\hline Código & Revisão & Documento & Tipo doc & Localização \\
\hline POP-ENF-001 & 11 & Assistência de Enfermagem Diante dos Efeitos Colaterais dos $\mathrm{CF}$ & POP & Enfermagem \\
\hline POP-ENF-043 & 02 & Controle e rastreabilidade dos artigos médicos hospitalares & POP & Enfermagem \\
\hline POP-ENF-003 & 14 & Recepção do Paciente no Ambulatónio de Quimioterapia & POP & Enfermagem \\
\hline POP-ENF-006 & 12 & Punção e Manutenção de Acesso Venoso Periférico & POP & Enfermagem \\
\hline POP-ENF-007 & 13 & Punção de Cateter Totalmente Implantado & POP & Enfermagem \\
\hline POP-ENF-004 & 12 & Vias de Administração de Drogas Antineoplásicas & POP & Enfermagem \\
\hline POP-ENF-011 & 11 & Extravasamento de Drogas Antineoplásicas & POP & Enfermagem \\
\hline POP-ENF-012 & 11 & Paramentação Durante Administração de Quimioterápicos & POP & Enfermagem \\
\hline POP-ENF-015 & 13 & Checagem da Prescrição Médica & POP & Enfermagem \\
\hline POP-ENF-016 & 12 & Administração e Controle do Oxigênio na Sala de Quimioterapia & POP & Enfermagem \\
\hline POP-ENF-017 & 11 & Monitorização Cardiaca e Oximetria de Pulso & POP & Enfermagem \\
\hline POP-ENF-018 & 11 & Atendimento a Parada Cárdio-Respiratória & POP & Enfermagem \\
\hline POP-ENF-019 & 13 & Conferência e Montagem da Sala de Emergência & POP & Enfermagem \\
\hline POP-ENF-023 & 12 & Anotação de Enfermagem e Passagem de plantão & POP & Enfermagem \\
\hline POP-ENF-026 & 11 & Sondagem Vesical & POP & Enfermagem \\
\hline POP-ENF-029 & 13 & Sinais Vitais e Escala Mews & POP & Enfermagem \\
\hline POP-ENF-030 & 11 & Realização de ECG & POP & Enfermagem \\
\hline POP-ENF-037 & 06 & Admissão do paciente pela Equipe de Enfermagem para Admini & POP & Enfermagem \\
\hline POP-ENF-036 & 04 & Retroalimentacao & POP & Enfermagem \\
\hline
\end{tabular}

Fig. 18: Lista de POPs. Fonte: Cedida pela instituição.

\section{Regimento interno da enfermagem}

É um instrumento gerencial normativo, de caráter flexível, que direciona e disciplina o serviço de enfermagem. Ele deverá ser atualizado anualmente ou sempre que houver mudanças. Ele contém um histórico de verificações e atualizações, capítulos e definições, conforme exemplo:

\begin{tabular}{|c|c|c|c|c|c|c|}
\hline & \multicolumn{6}{|c|}{$\begin{array}{c}\text { REGIMENTO INTERNO DO SERVIÇO } \\
\text { DO ENEERMAGEM }\end{array}$} \\
\hline \multicolumn{7}{|c|}{ 1. CONTROLE HISTÓRICO } \\
\hline Revisao & Data & \begin{tabular}{|c|}
$\mathbf{N}^{\circ}$ de \\
páginas
\end{tabular} & Historico & Elaboraçao & Verntıcaçao & Aprovaçao \\
\hline$\infty$ & $24 / 04 / 12$ & 12 & $\begin{array}{l}\text { Emizabo } \\
\text { Inicial }\end{array}$ & Emissōo Inicial & Lilion Loge & Cibele Antunes \\
\hline 01 & $10 / 10 / 14$ & 13 & Revisōo & Lilian Loge & Lilian Pimenta & Cibele Antunes \\
\hline 02 & $19 / 11 / 2018$ & 13 & Revisōo & Whorigns sontes. & $\begin{array}{c}\text { Aline } \\
\text { Mogainöes }\end{array}$ & Cibele Antunes \\
\hline$c 3$ & $06 / 04 / 2020$ & 23 & Revisōo & Bugrigngsantes. & Luciana Soarea & Luiz Adelmo begli \\
\hline \multicolumn{7}{|c|}{ CAPITULO I } \\
\hline \multicolumn{7}{|c|}{ DA DEFINIC̣ĀO: } \\
\hline
\end{tabular}

Fig. 19: Regimento interno da enfermagem. Fonte: Cedida pela instituição. 


\section{RECIMA21 - REVISTA CIENTÍFICA MULTIDISCIPLINAR}

DIAGNÓSTICO SITUACIONAL DE UMA CLÍNICA DE TRATAMENTO ONCOLÓGICO DE BELO HORIZONTE Alice Edwirges de Castro Andrade, Daniel dos Santos Fernandes, Aline Corrêa de Almeida, Eliada Neves de Queiroz Abreu, Nayara Pereira Mota, Nicoly Cirino de Rezende, Tailane de Jesus Santos

\begin{tabular}{|l|l|l|l|l|l|}
\hline Código & Revisão & Documento & Tipo doc & Localização \\
\hline PRO-CCO-001- & Protocolo Multidisciplinar de Manejo de Fadiga & Protocolo & Equipe Interdisciplir \\
\hline PRO-CCO-002 & Protocolo Multidisciplinar de Manejo de Náuseas e Vômitos & Protocolo & Equipe Interdisciplir \\
\hline PRO-CCO-003 & Protocolo Multidisciplinar de Manejo de Neutropenia & Protocolo & Equipe Interdisciplir \\
\hline PRO-CCO-004 & Protocolo Multidisciplinar de Manejo de Toxicidade Cutânea (Ri Protocolo & Equipe Interdisciplir \\
\hline PRO-CCO-005 & Protocolo Multidisciplinar de Manejo de Mucosite & Protocolo & Equipe Interdisciplir \\
\hline PRO-CCO-006 & Protocolo Multidisciplinar de Manejo de Neuropatia Periférica & Protocolo & Equipe Interdisciplir \\
\hline PRO-CCO-007 & Protocolo Multidisciplinar de Manejo de Cardiotoxicidade & Protocolo & Equipe Interdisciplir \\
\hline PRO-CCO-008 & Protocolo Multidisciplinar de Manejo de Trombocitopenia & Protocolo & Equipe Interdisciplir \\
\hline PRO-CCO-009 & Protocolo Multidisciplinar de Manejo de Anemia & Protocolo & Equipe Interdisciplir \\
\hline PRO-CCO-010 & Protocolo Multidisciplinar de Manejo de Eventos Tromboembóli Protocolo & Equipe Interdisciplir \\
\hline PRO-CCO-011 & Protocolo Multidisciplinar de Manejo de Dificuldade na Diarreia Protocolo & Equipe Interdisciplir \\
\hline PRO-CCO-012 & Protocolo Multidisciplinar de Manejo de Anorexia & Protocolo & Equipe Interdisciplir \\
\hline PRO-CCO-013 & Protocolo Multidisciplinar de Manejo de Fogachos & Protocolo & Equipe Interdisciplir \\
\hline PRO-CCO-014 & Protocolo Multidisciplinar de Manejo de Síndrome Fibromiálgice Protocolo & Equipe Interdisciplir \\
\hline PRO-CCO-015 & Protocolo Multidisciplinar de Manejo de Constipação Intestinal & Protocolo & Equipe Interdisciplir \\
\hline PRO-CCO-016 & Protocolo Multidisciplinar de Manejo de Insuficiência Renal & Protocolo & Equipe Interdisciplir \\
\hline PRO-CCO-017 & Protocolo Multidisciplinar de Manejo de Alopécia & Protocolo & Equipe Interdisciplir \\
\hline
\end{tabular}

Fig. 21: Lista de Protocolos. Fonte: Cedida pela instituição.

\begin{tabular}{|c|c|c|c|c|c|c|c|c|}
\hline \multicolumn{9}{|c|}{$\begin{array}{l}\text { Planilha de Verificação e Segurança de Equipamentos / Aparelhos Carrinho de Emergência } \\
\text { Mês: Dezembro/2020 }\end{array}$} \\
\hline Data & $\begin{array}{c}\text { Desfibriladore } \\
\text { Monitor,Cardiaco }\end{array}$ & $\begin{array}{c}\text { Oximetro de } \\
\text { Pulso }\end{array}$ & Aspirador & Laringoscopio & $\begin{array}{l}\text { Balas de oxigąnio } \\
\text { Fluxópzatral } \\
\text { Nanometro }\end{array}$ & $\begin{array}{l}\text { Validade da pilha } \\
\text { lasinga (data) }\end{array}$ & $\begin{array}{c}\mathrm{N}^{\circ} \\
\text { do Lacre }\end{array}$ & Colaborador \\
\hline & $\begin{array}{c}\text { Alarne / choque / } \\
\text { bateria }\end{array}$ & $\begin{array}{c}\text { Bateria e } \\
\text { Infravermelho }\end{array}$ & Suç̧âo & $\begin{array}{l}\text { Lâmpadas } \\
\text { Oxidaş̧ấ }\end{array}$ & - & - & - & - \\
\hline & & & & & & & & \\
\hline & & & & & & & & \\
\hline & & & & & & & & \\
\hline & & & & & & & & \\
\hline & & & & & & & & \\
\hline & & & & & & & & \\
\hline & & & & & & & & \\
\hline & & & & & & & & \\
\hline & & & & & & & & \\
\hline & & & & & & & & \\
\hline & & & & & & & & \\
\hline & & & & & & & & \\
\hline & & & & & & & & \\
\hline & & & & & & & & \\
\hline & & & & & & & & \\
\hline & & & & & & & & \\
\hline & & & & & & & & \\
\hline & & & & & & & & \\
\hline & & & & & & & & \\
\hline \begin{tabular}{|l} 
Nome \\
Equip:
\end{tabular} & 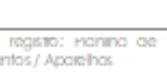 & & \begin{tabular}{l|l|l|l|l}
0 &
\end{tabular} & 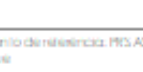 & & & 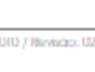 & \\
\hline
\end{tabular}

Fig. 22: Modelo de planilha de controle. Fonte: Cedida pela instituição. 


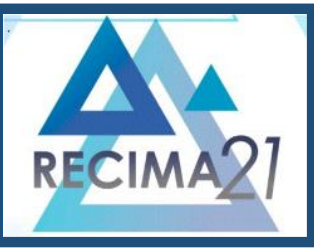

\section{RECIMA21 - REVISTA CIENTÍFICA MULTIDISCIPLINAR}

DIAGNÓSTICO SITUACIONAL DE UMA CLÍNICA DE TRATAMENTO ONCOLÓGICO DE BELO HORIZONTE Alice Edwirges de Castro Andrade, Daniel dos Santos Fernandes, Aline Corrêa de Almeida, Eliada Neves de Queiroz Abreu, Nayara Pereira Mota, Nicoly Cirino de Rezende, Tailane de Jesus Santos

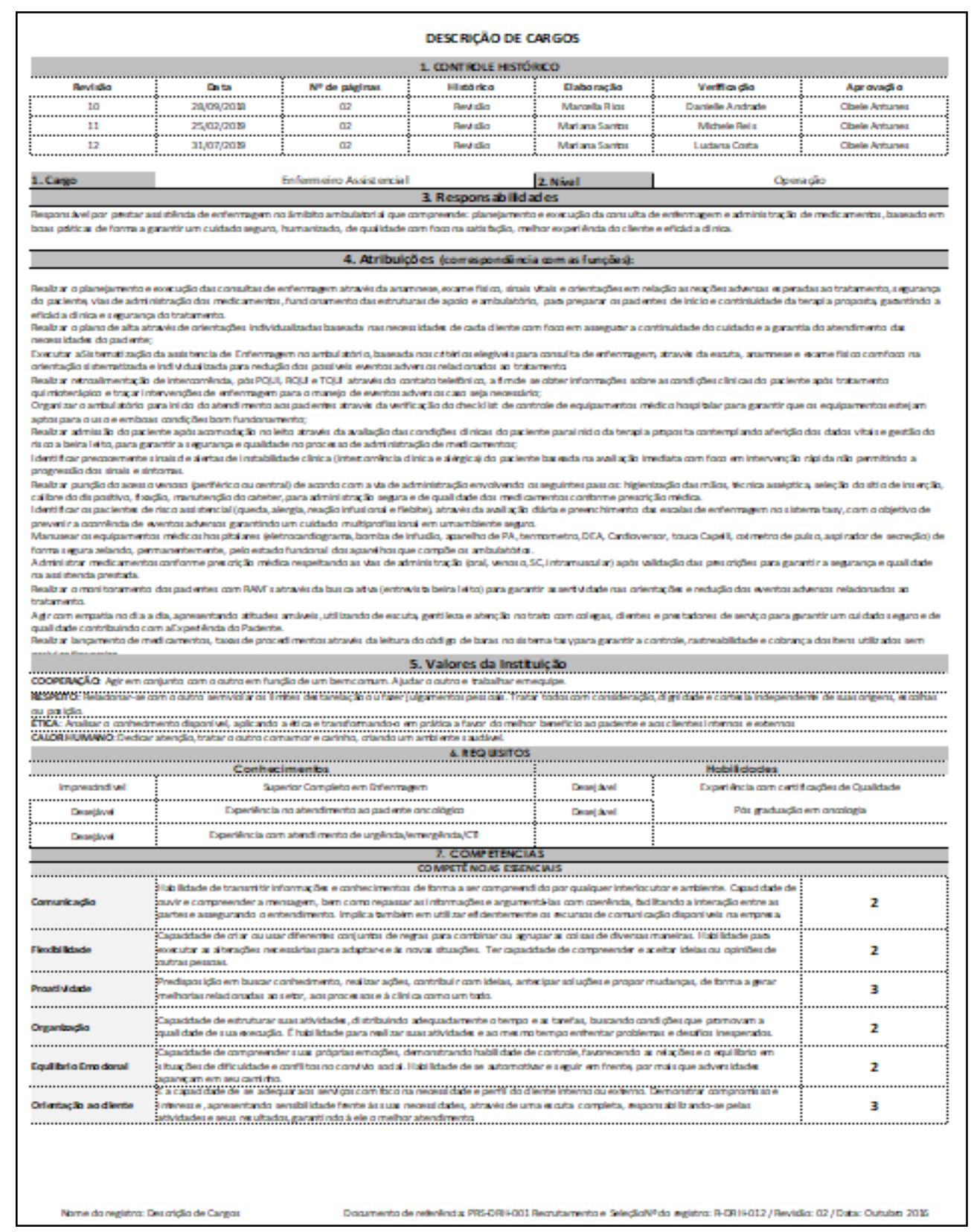

Fig. 23: Descrição de cargos. Fonte: Cedida pela instituição.

\section{Mapas de controle e checklists}

Os checklists e mapas de controle desempenham função na gestão de risco e segurança do paciente e profissionais. Eles fornecem dados de forma dinâmica e rápida para um trabalho preventivo e para ações em situações inesperadas dentro do ambiente de saúde. 


\section{RECIMA21 - REVISTA CIENTÍFICA MULTIDISCIPLINAR}

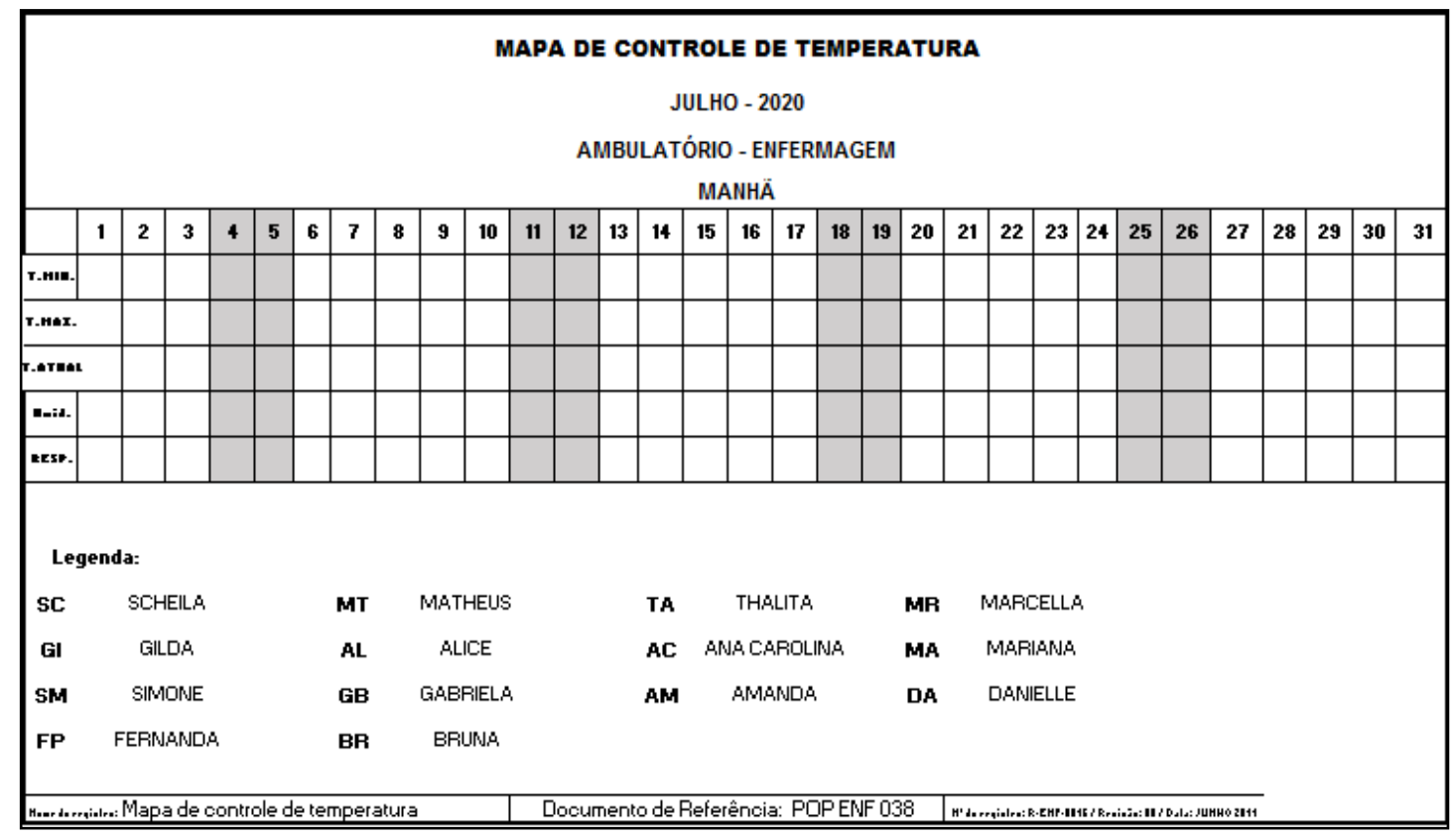

Fig. 24: Mapa de controle de temperatura. Fonte: Cedida pela instituição.

\begin{tabular}{|c|c|c|c|c|c|c|}
\hline \multirow[b]{2}{*}{ Material } & \multicolumn{6}{|c|}{ Formulário de Check list do ambulatório de quimioterapia } \\
\hline & RuantidadeValidade & 1 & $\begin{array}{l}\text { Data } \\
1 \quad 1\end{array}$ & $\begin{array}{l}\text { Data } \\
1 \quad 1\end{array}$ & $\begin{array}{l}\text { Data } \\
1 \quad 1\end{array}$ & $\begin{array}{l}\text { Data } \\
11\end{array}$ \\
\hline \multicolumn{7}{|l|}{ Material de uso coletivo } \\
\hline Aparelho de pressẫo + estetoscópio & 3 & & & & & \\
\hline Bomba de infusẫo (testar) & 10 & & & & & \\
\hline Equipamento touca Capelli & 1 & & & & & \\
\hline Bala de oxigênio Média & 1 & & & & & \\
\hline Maleta de emergência & 1 & & & & & \\
\hline Glicosimetro & 1 & & & & & \\
\hline Palm & 3 & & & & & \\
\hline Macas com colchỗes (1cada) & 2 & & & & & \\
\hline Cadeiras de rodas (2-obesos) & 5 & & & & & \\
\hline Carrinho medicamento & 5 & & & & & \\
\hline Spot & 3 & & & & & \\
\hline Responsável pelo check list & & Ass. Carimbo & Ass. Carimbo & Ass. Carimb & Ass. Carimb & Ass. Carimb \\
\hline Nome registro: Check list do ambulató & nioterapia & & Documentod & le referência: & POP & \\
\hline
\end{tabular}

Fig. 25: Formulário de checklist do ambulatório de quimioterapia. Fonte: Cedida pela instituição. 


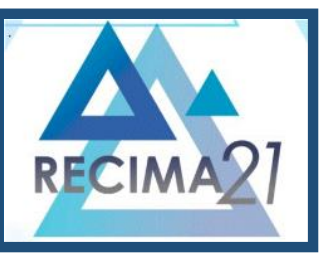

\section{RECIMA21 - REVISTA CIENTÍFICA MULTIDISCIPLINAR}

DIAGNÓSTICO SITUACIONAL DE UMA CLÍNICA DE TRATAMENTO ONCOLÓGICO DE BELO HORIZONTE Alice Edwirges de Castro Andrade, Daniel dos Santos Fernandes, Aline Corrêa de Almeida, Eliada Neves de Queiroz Abreu, Nayara Pereira Mota, Nicoly Cirino de Rezende, Tailane de Jesus Santos

Escalas de enfermagem

São documentos de comprovação de dimensionamento de pessoal e de horas de enfermagem disponibilizadas para os pacientes. É um documento realizado cotidianamente em todo o serviço.

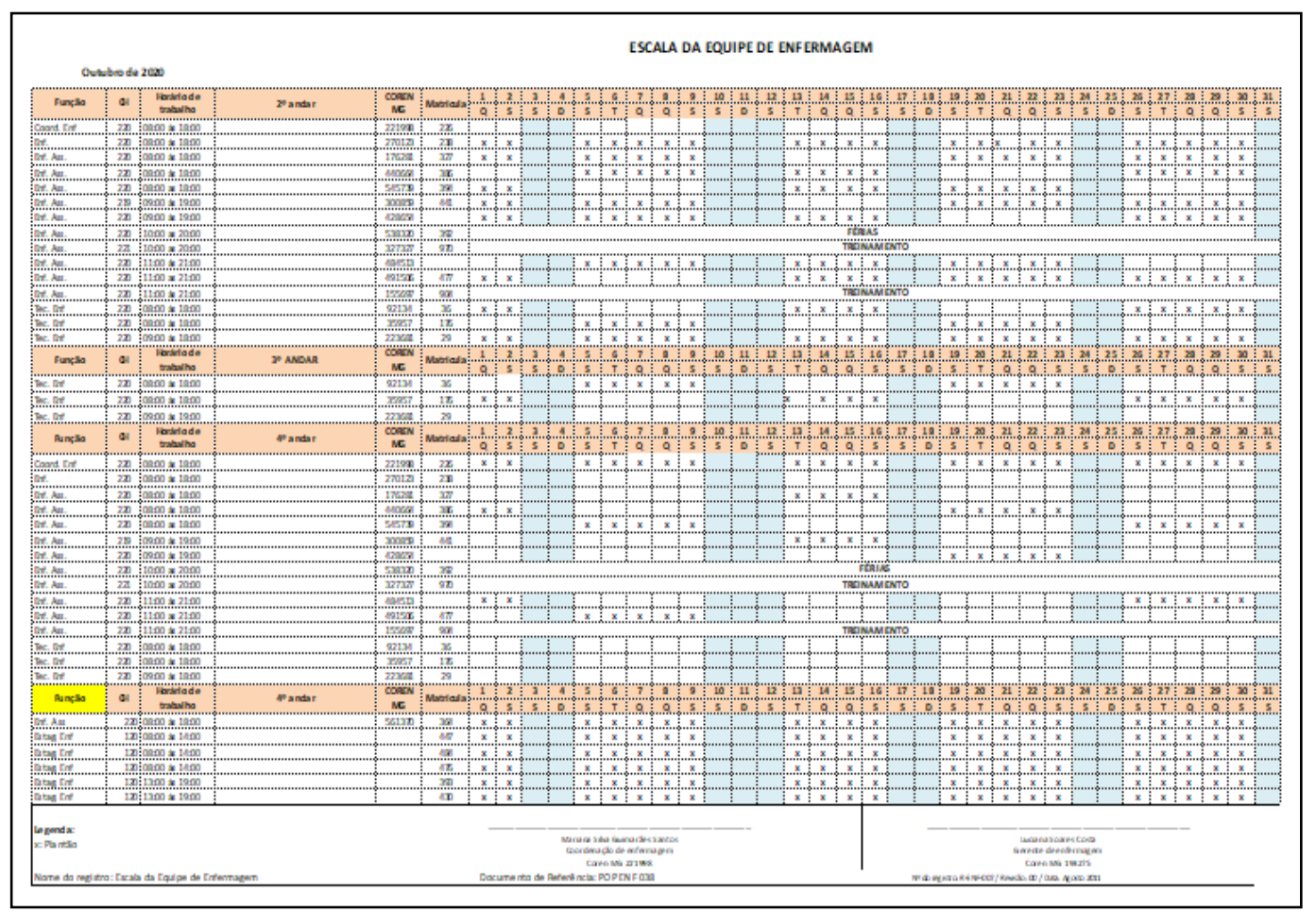

Fig. 26: Escala da Equipe de Enfermagem. Fonte: Cedida pela instituição.

\begin{tabular}{|c|c|c|c|c|c|c|c|c|c|c|c|}
\hline \multicolumn{12}{|c|}{$\begin{array}{l}\text { E SCALA DISTRIBUIÇĀO DE LEITOS } 2^{\circ} \text { ANDAR } \\
\text { Semana } 31 / 08 \text { a 04/09 }\end{array}$} \\
\hline $\begin{array}{l}\text { Funcionáriol } \\
\text { Hora }\end{array}$ & $8: 00$ as $9: 00 \mid$ & $9.00 \approx 10.00$ & 10.0021100 & $1100 \approx 12000$ & 12002513000 & $13.00 \mathrm{a}=14.00$ & $14.00 \approx 15.00$ & \begin{tabular}{|l|l|}
15000 & 18000
\end{tabular} & $18.00 \approx 19.00$ & $\mid 19000200000$ & $20000 \approx 21.00$ \\
\hline & & $\mid \begin{array}{ll}\operatorname{NN} 3 \\
\end{array}$ & $\mid \mathbb{N} J 3$ & $\mid \mathrm{IN} / 3$ & & $\mathbb{I N J} 3$ & $\mid \mathbb{N} J 3$ & \begin{tabular}{|l|l|}
$\operatorname{IN} 3$ & \\
\end{tabular} & \begin{tabular}{|l|l|} 
INJ 3 \\
\end{tabular} & & \\
\hline & & \begin{tabular}{|l|l|} 
Leito 1 a 5 \\
\end{tabular} & Leito 1 a 5 & \begin{tabular}{|l|} 
Leito 1 a 5 \\
\end{tabular} & Leito 1 a 5 & & \begin{tabular}{|l|} 
Leito 1 a 5 \\
\end{tabular} & Leit 1 a 5 & Leit 1 a 5 & & \\
\hline & & & & Leito $10 \mathrm{a} 13$ & Leito 10 a 13 & Léto 10 a 13 & & Leilo 10 a 13 & Leins 10 a 13 & 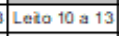 & \begin{tabular}{|l|l|} 
Leito 10 a 13 \\
\end{tabular} \\
\hline & & & $\mathbb{I N J} 3$ & \begin{tabular}{|l|} 
IN 3 \\
\end{tabular} & IN 3 & & $\mathbb{N N J 3}$ & IN 3 & $\mid \mathbb{N} J 3$ & $\mid \mathbb{N} N{ }^{\prime} 3$ & \\
\hline & \multicolumn{11}{|c|}{ Férias } \\
\hline & & & & & & & & & & & \\
\hline & $\operatorname{INN} 3$ & $\operatorname{INN} 3$ & $\mathbb{N N J 3}$ & & $\operatorname{INN} 3$ & $\mathbb{1 N J} 3$ & $\mathbb{N N J 3}$ & $\operatorname{INN} 3$ & & & \\
\hline & \begin{tabular}{|l|l|} 
& Leíto 14 a 17 \\
\end{tabular} & \begin{tabular}{|l|l|}
$L e i b$ & 14 a 17 \\
\end{tabular} & Leato 14 a 17 & Leiio 14 a 17 & & Leita 14 a 17 & \begin{tabular}{|l|l|} 
\\
\end{tabular} & Leilio 14 a 17 & & & \\
\hline & & & & & & & & & & & \\
\hline & & & Leăo 10 a 13 & 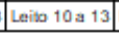 & \begin{tabular}{|l|l|} 
\\
\end{tabular} & & Leto 10 a 13 & Leilo 10 a 13 & L Lish 10 a 13 & Leto 10 a 13 & \\
\hline \multicolumn{11}{|c|}{ ESCALA DISTRIBUIÇÄO DE LEITOS $4^{\circ}$ ANDAR } & \\
\hline $\begin{array}{c}\text { Funcionáriol } \\
\text { Hora }\end{array}$ & 8.00 as $9: 00$ & $9: 00 \approx 10.00$ & 10.002 s 11.00 & $1100=12000$ & 1200241300 & $1300 \approx 14.00$ & $1400=15000$ & $1500 \mathrm{a} / 1800$ & $18.00 \Omega$ s 19.00 & . $\mid 1900 \approx 200000$ & \\
\hline & & & & & & & & & & & \\
\hline & & & & & & & & & & & \\
\hline & & & APOIO & APOIO & APOIO & & APOIO & APOIO & APOIO & APOIO & \\
\hline & & & & Leíio 18 a a 22 & Leito 18 a 22 & Léto 18 a 22 & & Leitio 18 a 22 & Leiso 18 a 22 & Leto 18 a 22 & \\
\hline & \multicolumn{10}{|c|}{ Férias } & \\
\hline & & & & & & & & & & & \\
\hline & & & & & & & & & & & \\
\hline & & & & & & & & & & & \\
\hline & \begin{tabular}{|l|} 
Leito 23 a 26 \\
\end{tabular} & Leiso 23 a 26 & Leta 23 a 26 & & Leî̉o 23 a 26 & Léito 23 a 26 & Leťo 23 a 26 & Leaito 23 a 26 & & & \\
\hline & & & & & & & & & & & \\
\hline
\end{tabular}

Fig. 27: Escala de distribuição dos leitos. Fonte: Cedida pela instituição. 


\section{RECIMA21 - REVISTA CIENTÍFICA MULTIDISCIPLINAR}

\section{IDENTIFICAÇÃO E DEFINIÇÃO DE INDICADORES ASSISTENCIAIS EM ENFERMAGEM}

Os indicadores assistenciais em enfermagem são essenciais para o acompanhamento, controle e avaliação de resultados de processos de trabalhos assistenciais e metas alcançadas. Com isso, é possível comparar o desempenho e qualidade de serviços prestados ao decorrer do tempo (COREN, 2020).

Estes indicadores apresentam medidas quantitativas e qualitativas dos cuidados prestados. Para sua construção é necessário que sejam elaborados e criticamente avaliados dentro de legislações e padrões das literaturas existentes.

Diante disso, a clínica dispõe dos seguintes indicadores:

\begin{tabular}{|c|l|}
\hline \multicolumn{2}{|c|}{ INDICADORES AMBULATÓRIO QT } \\
\hline $\mathbf{1}$ & Taxa de ocupação \\
\hline $\mathbf{2}$ & SLA tempo de espera enfermagem \\
\hline $\mathbf{3}$ & Taxa de deterioração clínica de pacientes no ambulatório \\
\hline $\mathbf{4}$ & Retroalimentação realizadas com sucesso dentro do prazo (3 a 5 dias) \\
\hline $\mathbf{7}$ & ADESÃO AO PROTOCOLO DE HIGIENIZAÇÃO DAS MÃOS \\
\hline $\mathbf{8}$ & Consumo de alcool gel por paciente dia \\
\hline $\mathbf{9}$ & Consumo de sabonete liquido por paciente dia \\
\hline $\mathbf{1 0}$ & Taxa de assertividade na administração de medicamentos \\
\hline $\mathbf{1 1}$ & Indice de Intercorrência clinica \\
\hline & \\
\cline { 2 - 2 } & \\
\hline $\mathbf{1}$ & Índice de Reação adversa medicamentosa \\
\hline $\mathbf{2}$ & Efetividade das barreiras de segurança assistencial Near Miss \\
\hline $\mathbf{3}$ & TAXA DE CONFORMIDADE NA TRANSIÇÃO DE CUIDADOS \\
\hline $\mathbf{4}$ & Taxa de segurança global \\
\hline
\end{tabular}

Fig. 28: Indicadores Ambulatório Quimioterapia. Fonte: Cedida pela instituição.

Os indicadores da figura 28 podem ser delineados por objetivo e meta, sendo possível reconhecer a sua importância na mensuração da qualidade da assistência prestada. A seguir, serão descritos cada um desse indicadores:

\section{- SLA tempo de espera enfermagem}

Objetivo: Monitorar o tempo de espera para início dos atendimentos aos pacientes em uso de protocolos venosos.

Meta: $80 \%$ dos atendimentos em até $20 \mathrm{~min}$.

Em SLA o indicador está relacionado à organização, clareza e fluidez no processo de comunicação entre profissionais, gerando maior qualidade no atendimento. 


\section{RECIMA21 - REVISTA CIENTÍFICA MULTIDISCIPLINAR}

- Taxa de deterioração clínica de pacientes no ambulatório

Objetivo: Monitorar o estado clinico do paciente durante a administração dos medicamentos (via endovenosa) através da avaliação dos parâmetros fisiológicos dos pacientes e classificação de MEWS.

Meta: Monitoramento

A relevância deste indicador está em avaliar e classificar o cliente dentro de um parâmetro chamado MEWS, onde ele pode ser categorizado dentro de:

MEWS 1: Não se aplica nenhum tipo de intervenção.

MEWS 2: É preciso comunicar supervisão de enfermagem e checar os dados vitais a cada 2 horas.

MEWS 3: É necessário comunicar ao médico de plantão e verificar os dados vitais a cada 30 minutos.

MEWS 4: O paciente já se encontra em estado mais grave e as medidas de intervenções são realizadas de acordo com a demanda e auxilio do médico.

- Retroalimentação realizadas com sucesso dentro do prazo (3 a 5 dias)

Objetivo: Garantir o monitoramento dos pacientes com reações adversas medicamentosas (RAM's), através do contato telefônico, após início do tratamento (primeira quimioterapia, retorno de quimioterapia e troca de quimioterapia) e pacientes cadastrados na Intellicare para manejo dos possíveis efeitos colaterais (prazo de 3 a 5 dias úteis após administração do medicamento e para intercorrência no ambulatório em até 24 horas úteis e Robô Caren somente pacientes com RAM's grau III e IV durante o tratamento).

Meta: $95 \%$

- Consumo de álcool gel por paciente dia

Objetivo: Avaliar o consumo de álcool gel para higienização das mãos.

Meta: $20 \mathrm{ml}$

Os responsáveis por mensurar esse consumo é o CCIH (Comissão de Controle de Infecção Hospitalar) ajudando a garantir que haja consumo e reposição desse material.

\section{- Consumo de sabonete líquido por paciente dia}

Objetivo: Avaliar o consumo de sabonete líquido para higienização das mãos.

Meta: $6 \mathrm{ml}$

O $\mathrm{CClH}$, assim como no consumo de álcool gel, também é o responsável pelo rastreamento do consumo de sabonete líquido, para controle de infecções.

\section{- Taxa de infecção da corrente sanguínea}




\section{RECIMA21 - REVISTA CIENTÍFICA MULTIDISCIPLINAR}

DIAGNÓSTICO SITUACIONAL DE UMA CLíNICA DE TRATAMENTO ONCOLÓGICO DE BELO HORIZONTE Alice Edwirges de Castro Andrade, Daniel dos Santos Fernandes, Aline Corrêa de Almeida, Eliada Neves de Queiroz Abreu, Nayara Pereira Mota, Nicoly Cirino de Rezende, Tailane de Jesus Santos

Objetivo: Controlar os dados epidemiológicos de infecção por sítios (cateter, sonda vesical de demora)

Meta: 0

Esse é um indicador para o controle de infecções, também feito pelo $\mathrm{CCIH}$. Para investigar se a infecção foi causada na clínica.

\section{- Taxa de conformidade na transição de cuidados}

Objetivo: Garantir a comunicação assertiva entre os profissionais de saúde na continuidade do cuidado.

Meta: $100 \%$

Este indicador ajuda a equipe de enfermagem no controle da comunicação na troca de plantões, evitando possíveis erros.

- Índice de reação adversa medicamentosa

Objetivo: Identificar as Reações Adversas Medicamentosas (RAM's) e aprimorar ações preventivas no manejo das reações.

Meta: Monitoramento

Esse indicador contabiliza o número de reações adversas medicamentosas que aconteceram, com intuito de aprimorar ações preventivas.

\section{- Taxa de assertividade na administração de medicamentos}

Objetivo: Garantir a administração correta e segura dos medicamentos.

Meta: $100 \%$

O indicador mensura a assertividade na administração dos medicamentos, que passa pelos "9 certos da medicação":

1. Paciente certo;

2. Medição certa;

3. Dosagem certa;

4. Via certa;

5. Hora certa;

6. Registro certo;

7. Abordagem certa;

8. Forma certa;

9. Monitoramento certo.

A conferência dos " 9 certos" antes do início dos procedimentos impacta de forma significativa na assistência, podendo evitar o erro. 


\section{RECIMA21 - REVISTA CIENTÍFICA MULTIDISCIPLINAR}

DIAGNÓSTICO SITUACIONAL DE UMA CLíNICA DE TRATAMENTO ONCOLÓGICO DE BELO HORIZONTE Alice Edwirges de Castro Andrade, Daniel dos Santos Fernandes, Aline Corrêa de Almeida, Eliada Neves de Queiroz Abreu, Nayara Pereira Mota, Nicoly Cirino de Rezende, Tailane de Jesus Santos

- Efetividade nas barreiras de segurança (Near Miss) - ENF, FAR e CCO

Objetivo: Monitorar a efetividade das barreiras de segurança utilizadas em todos os processos assistenciais.

Meta: $100 \%$

\section{- Taxa de segurança global}

Objetivo: Monitorar todos os eventos adversos na instituição.

Meta: $100 \%$

Esse indicador relaciona-se ao número de intercorrências que podem acontecer durante um período de plantão da equipe de enfermagem auxiliando na mensuração de possíveis inconsistências de ações de assistência de enfermagem.

\section{- Índice de intercorrência clínica}

Objetivo: Monitorar os pacientes que apresentaram intercorrência clínica e que necessitaram de transferência hospitalar.

Meta: Monitoramento

\section{- Índice de queda}

Objetivo: Monitorar e promover a segurança do paciente na prevenção de quedas.

Meta: 0

O índice de queda é monitorizado de acordo com a condição do paciente. Esse processo inicia-se quando o paciente chega à clínica, onde será identificado com uma pulseira de cor azul, em caso indicativo de risco de queda elevado.

\section{CONSIDERAÇÕES FINAIS}

O diagnóstico situacional foi elaborado para conhecer a instituição, dos pontos positivos aos de melhoria, identificando os principais fatores envolvidos nesse processo.

Ao reconhecer a estrutura organizacional foi possível identificar como a instituição de organiza, quanto aos cargos e funções, estabelecendo a forma como ela será hierarquizada. Quanto aos recursos físicos e infraestrutura foi possível avaliar se a instituição se adequa aos requisitos da Anvisa. Foi identificado apenas uma divergência, no caso, a distância mínima entre os leitos.

Os recursos humanos avaliados demonstraram corresponder às exigências do Conselho Federal de Enfermagem, apresentando uma escala de enfermagem adequada ao serviço prestado. É possível identificar uma divergência na quantidade de técnicos de enfermagem, que é inferior. Porém, também há uma quantidade superior a exigida em relação aos enfermeiros. Tratando-se de um serviço com maior número de procedimentos privativos ao enfermeiro, a diferença torna-se compensatória. 


\section{RECIMA21 - REVISTA CIENTÍFICA MULTIDISCIPLINAR}

$\mathrm{Na}$ avaliação dos recursos materiais, a instituição de oncologia apresenta métodos de barreiras que auxiliam e proporcionam o bom desempenho e a qualidade exercidas pelos profissionais da saúde. Durante a execução de suas atividades observa-se a realização da identificação do paciente com pulseira, identificação dos medicamentos, armazenamentos em locais apropriados e identificados, dupla checagem entre profissionais e com o paciente a beira leito e utilização de aparelho eletrônico realizando a conferência por código de barra existentes na pulseira de identificação e rótulo do medicamento.

Apesar dos controles e processos estabelecidos pela instituição e gestores, observa-se falha no processo de finalização de cobrança de materiais da maleta de emergência pela equipe de enfermagem. Ao deixar de realizar a cobrança imediata na conta do paciente, observa-se a perda da rastreabilidade, além da existência de divergências no estoque da maleta de emergência no posto de enfermagem e também no estoque de controle pela farmácia. A reposição dos materiais da maleta de emergência pela farmácia poderá ser comprometida e a falta de cobrança poderá gerar glosa da conta pelo convênio, ocasionando risco financeiro.

Com o diagnóstico situacional, foi possível identificar, também, os instrumentos gerenciais utilizados pela instituição. Eles auxiliam em toda a gestão da instituição e apresentam-se assertivos em sua função.

A instituição também dispõe de diversos indicadores assistenciais, que tornam a assistência mais efetiva e de qualidade. Eles são pautados por objetivos e metas, tornando, assim, a assistência mais qualificada e controlada.

O diagnóstico situacional proporcionou a identificação e avaliação dos pontos fortes e de melhoria da empresa. No caso, os pontos fortes se sobressaem, mostrando como a instituição se preocupa com a qualidade do serviço prestado, colaboradores e pacientes.

Portanto, após todo esse processo de identificação e avaliação para a construção do diagnóstico situacional dessa clínica oncológica de Belo Horizonte, é possível reconhecer a importância do desenvolvimento dessas habilidades em acadêmicos de enfermagem. Todo o processo de coleta, identificação e avaliação de dados proporciona ao acadêmico a capacidade de senso crítico, gestão e de uma assistência assertiva.

\section{REFERÊNCIAS BIBLIOGRÁFICAS}

ALMEIDA, Maria de Lourdes de; SEGUI, Maria Luíza Hexsel; MAFTUM, Mariluci Alves; LABRONICI, Liliana Maria; PERES, Aida Maris. Instrumentos gerenciais utilizados na tomada de decisão do enfermeiro no contexto hospitalar. Texto \& Contexto - Enfermagem, v. 20, p. 131-137, 2011. Disponível em: https://www.scielo.br/scielo.php?script=sci arttext\&pid=S010407072011000500017\&lng=pt\&tlng=pt. Acesso em: 20 nov. 2020.

AMORIM, Gláucia Maria; QUINTÃO, Eliana Cardoso Vieira; MARTELLI JÚNIOR, Hercílio; BONAN, Paulo Rogério Ferreti. Prestação de serviços de manutenção predial em Estabelecimentos 
DIAGNÓSTICO SITUACIONAL DE UMA CLÍNICA DE TRATAMENTO ONCOLÓGICO DE BELO HORIZONTE Alice Edwirges de Castro Andrade, Daniel dos Santos Fernandes, Aline Corrêa de Almeida, Eliada Neves de Queiroz Abreu, Nayara Pereira Mota, Nicoly Cirino de Rezende, Tailane de Jesus Santos

Assistenciais de Saúde. Ciência \& Saúde Coletiva, v. 18, n. 1, p. 145-158, jan. 2013. Disponível em: https://www.scielo.br/scielo.php?script=sci arttext\&pid=S141381232013000100016\&lng=en\&nrm=iso \&tlng=en. Acesso em: 15 nov. 2020.

ANVISA. Aspectos da segurança no ambiente hospitalar. Brasília: ANVISA, 2003. Disponível em: http://www.anvisa.gov.br/servicosaude/manuais/manual seg hosp.htm. Acesso em: 21 nov. 2020.

ANVISA. Resolução no 220, de 21 de setembro de 2004. Dispõe sobre as condições para a promoção, proteção e recuperação da saúde como direito fundamental do ser humano; considerando os riscos inerentes à terapia antineoplásica a que fica exposto o paciente que se submete a tais procedimentos. $\quad$ Brasília: $\quad$ ANVISA, $2004 . \quad$ Disponível em: file:///C:/Users/FUN\%20MACHINE/Downloads/RDC n 220 de 2004 Terapia Antineoplsica.pdf.

Acesso em: 12 nov. 2020.

ANVISA. Resolução no 50, de $\mathbf{2 1}$ de fevereiro de 2002. Dispõe sobre o regulamento técnico para planejamento, programação, elaboração e avaliação de projetos físicos de estabelecimentos assistenciais de saúde. Brasília: ANVISA, 2002. Disponível em: file:///C:/Users/FUN\%20MACHINE/Downloads/RDC 2002-50.pdf. Acesso em: 10 nov. 2020.

BRASIL. Lei no 7.498, de 25 de junho de 1986. Dispõe sobre a regulamentação do exercício da Enfermagem e dá outras providências. Brasília: Legislação para o Exercício da Enfermagem, 1986. 6 p. Disponível em: http://www.cofen.gov.br/lei-n-749886-de-25-de-junho-de-1986 4161.html. Acesso em: 02 de maio 2020.

COFEN. Assessoria de Planejamento e Gestão. Conselho Federal de Enfermagem: Atribuições das Unidades Funcionais do Cofen. COFEN, Brasília/DF, v. 3, n. 18, p. 1-123, nov. 2020.

COFEN. Resolução no $\mathbf{0 5 4 3}$ de 2017. Atualiza e estabelece parâmetros para o dimensionamento do quadro de profissionais de enfermagem nos serviços/locais em que são realizadas atividades de enfermagem. Brasília: COFEN, 2017. Disponível em: http://www.cofen.gov.br/wpcontent/uploads/2017/05/RESOLU\%C3\%87\%C3\%830-COFEN-N\%C2\%BA-543-2017-completa.pdf.

Acesso em: 06 dez. 2020.

COFEN. Resolução no 45 de 12 de março de 2003. Dispõe sobre o regulamento técnico de boas práticas de utilização das soluções parenterais (SP) em Serviços de Saúde. São Paulo: COFEN, 2003. Disponível em: http://www.cofen.gov.br/resoluo-rdc-n-45-de-12-de-maro-de-2003 4323.html. Acesso em: 21 nov. 2020.

COFEN. Resolução no 487, de 01 de setembro de 2015. Veda aos profissionais de enfermagem o cumprimento de prescrição médica à distância fornecida por meio de rádio, telefones fixos e/ou móveis, mensagem de sms (Short Message Service), correio eletrônico, redes sociais de internet ou quaisquer outros meios onde não conste o carimbo e assinatura do médico. São Paulo: COFEN, 2015. Disponível em: http://www.cofen.gov.br/resolucao-cofen-no-4872015 33939.htm. Acesso em: 21 nov. 2020.

COREN-MG. Plano de trabalho do Enfermeiro: subsídios para a realização do Diagnóstico Situacional do Serviço de Enfermagem e proposição de plano de ação estratégico. Belo Horizonte: Conselho Regional de Enfermagem-Coren-MG, 2020.

COREN-SE. Normas e rotinas. Aracaju: Conselho Regional de Enfermagem-Coren-SE, 2017. Disponível em: http://se.corens.portalcofen.gov.br/wp-content/uploads/2017/02/MODELO-NORMASROTINAS-E-POP.pdf. Acesso em: 01 dez. 2020.

DTIC/COFEN. As Categorias Profissionais da Enfermagem. Disponível em: http://biblioteca.cofen.gov.br/as-categorias-profissionais-da-enfermagem/. Acesso em: 14 nov. 2020. 


\section{RECIMA21 - REVISTA CIENTÍFICA MULTIDISCIPLINAR}

DIAGNÓSTICO SITUACIONAL DE UMA CLÍNICA DE TRATAMENTO ONCOLÓGICO DE BELO HORIZONTE Alice Edwirges de Castro Andrade, Daniel dos Santos Fernandes, Aline Corrêa de Almeida, Eliada Neves de Queiroz Abreu, Nayara Pereira Mota, Nicoly Cirino de Rezende, Tailane de Jesus Santos

DUTRA, Herica Silva; SANHUDO, Nádia Fontoura. Gerência em Enfermagem. Juiz de Fora: Universidade Federal de Juiz de Fora, 2019. Disponível em: https://www.ufjf.br/admenf/files/2019/03/Aula-1-Gere\%CC\%82ncia-em-Enfermagem-.pdf. Acesso em: 20 nov. 2020.

INTERACTIVE, Lookmysite. Equipe multidisciplinar para tratamento do câncer. Disponível em: http://www.oncoguia.org.br/conteudo/equipe-multidisciplinar/8213/50/. Acesso em: 22 nov. 2020.

MOURA, Bárbara Laisa Alves; CUNHA, Renata Castro da; FONSECA, Ana Carla Freitas. et al. Atenção primária à saúde: estrutura das unidades como componente da atenção à saúde. Revista Brasileira de Saúde Materno Infantil, v. 10, n. 1, p. 69-81, nov. 2010. Disponível em: https://www.scielo.br/scielo.php?script=sci arttext\&pid=S1519-38292010000500007. Acesso em: 15 nov. 2020.

ASSOCIAÇÃO BRASILEIRA DE NORMAS TÉCNICAS. ABNT NBR 5462:2015 -Trata a Respeito dos principais conceitos e terminologias que rodeiam a confiabilidade e mantenabilidade e dentre esses conceitos estão os tipos de manutenção. Disponível em: https://pt.slideshare.net/eavargas2512/nbr5462-2. Acesso em: 21 nov. 2020.

SANTOS, Luana Caroline dos. (Org.). Diagnóstico Situacional da Unidade Básica de Saúde Barreiro de Cima. Belo Horizonte: Universidade Federal de Minas Gerais, 2010. Disponível em 